\title{
Increased apolipoprotein C3 drives cardiovascular risk in type 1 diabetes
}

\author{
Jenny E. Kanter, ${ }^{1}$ Baohai Shao, ${ }^{1}$ Farah Kramer, ${ }^{1}$ Shelley Barnhart,, Masami Shimizu-Albergine, ${ }^{1}$ Tomas Vaisar, ${ }^{1}$ Mark J. Graham, ${ }^{2}$ \\ Rosanne M. Crooke, ${ }^{2}$ Clarence R. Manuel, ${ }^{3}$ Rebecca A. Haeusler, ${ }^{3}$ Daniel Mar, ${ }^{4}$ Karol Bomsztyk, ${ }^{4}$ John E. Hokanson, ${ }^{5}$ \\ Gregory L. Kinney, ${ }^{5}$ Janet K. Snell-Bergeon, ${ }^{6}$ Jay W. Heinecke, ${ }^{1}$ and Karin E. Bornfeldt ${ }^{1,7}$

\begin{abstract}
DDepartment of Medicine, UW Medicine Diabetes Institute, University of Washington, Seattle, Washington, USA. ${ }^{2}$ Ionis Pharmaceuticals, Carlsbad, California, USA. ${ }^{3}$ Department of Pathology and Cell Biology, Columbia University, New York, New York, USA. ${ }^{4}$ Department of Medicine, Division of Allergy and Infectious Disease, University of Washington, Seattle, Washington, USA. ${ }^{5}$ Department of Epidemiology, Colorado School of Public Health, University of Colorado Denver, Aurora, Colorado, USA. ${ }^{6}$ Barbara Davis Center for Diabetes, School of Medicine, University of Colorado Denver, Aurora, Colorado, USA. ${ }^{7}$ Department of Pathology, University of Washington, Seattle, Washington, USA.
\end{abstract}

\begin{abstract}
Type 1 diabetes mellitus (T1DM) increases the risk of atherosclerotic cardiovascular disease (CVD) in humans by poorly understood mechanisms. Using mouse models of T1DM-accelerated atherosclerosis, we found that relative insulin deficiency, rather than hyperglycemia, elevated levels of apolipoprotein C3 (APOC3), an apolipoprotein that prevents clearance of triglyceride-rich lipoproteins (TRLs) and their remnants. We then showed that serum APOC3 levels predict incident CVD events in subjects with T1DM in the Coronary Artery Calcification in Type 1 Diabetes (CACTI) study. To explore underlying mechanisms, we examined the impact of Apoc3 antisense oligonucleotides (ASOs) on lipoprotein metabolism and atherosclerosis in a mouse model of T1DM. Apoc3 ASO treatment abolished the increased hepatic expression of Apoc3 in diabetic mice, resulting in lower levels of TRLs, without improving glycemic control. APOC3 suppression also prevented arterial accumulation of APOC3-containing lipoprotein particles, macrophage foam cell formation, and accelerated atherosclerosis in diabetic mice. Our observations demonstrate that relative insulin deficiency increases APOC 3 and that this results in elevated levels of TRLs and accelerated atherosclerosis in a mouse model of T1DM. Because serum levels of APOC3 predicted incident CVD events in the CACTI study, inhibition of APOC3 might reduce CVD risk in patients with T1DM.
\end{abstract}

\section{Introduction}

Atherosclerotic cardiovascular disease (CVD) is a major cause of morbidity and mortality in subjects with type 1 diabetes mellitus (T1DM) (1). Although some results suggest that CVD risk associated with diabetes is declining in parallel with the reduced rate of CVD in the general population (2), the overall CVD risk remains higher in T1DM patients, especially in premenopausal women, in subjects with suboptimally controlled blood glucose levels, and in subjects with features of insulin resistance and metabolic syndrome (3).

The Diabetes Control and Complications Trial (DCCT), a prospective study with a mean follow-up of 6.5 years for microvascular and macrovascular disease in patients with T1DM, and its observational follow-up study, the Epidemiology of Diabetes Interventions and Complications (EDIC) study arm, demonstrated that improved glycemic control by intensive insulin therapy is associated with long-

\section{Related Commentary: p. 4074}

\footnotetext{
Conflict of interest: MJC and RMC are employed by lonis Pharmaceuticals and provided antisense oligonucleotides for the study. KEB and JEK have received research support from Novo Nordisk A/S. KB is a cofounder of and equity holder in Matchstick Technologies Inc., which has licensed the PIXUL technology from the University of Washington.

Copyright: @ 2019, American Society for Clinical Investigation

Submitted: January 21, 2019; Accepted: July 3, 2019; Published: August 26, 2019

Reference information: J Clin Invest. 2019;129(10):4165-4179.

https://doi.org/10.1172/JCl127308.
}

term reductions in mortality and cardiovascular events (4-6). Moreover, improved plasma lipids and lipoproteins appeared to mediate a substantial proportion of the protective effect of more stringent glucose control through intense insulin therapy over time (7).

Lowering LDL cholesterol (LDL-C) with statins is the best strategy documented to date for decreasing CVD risk in T1DM, but substantial residual risk remains. The increased risk is often attributed to hyperglycemia, fluctuating glucose levels, or glucosemediated metabolic memory $(8,9)$. However, several lines of evidence suggest that glucose does not directly account for the increased CVD risk in patients with diabetes, or at least is not the only risk factor $(7,10,11)$. It is likely that relative insulin deficiency or insulin resistance could explain at least some of the residual CVD risk associated with T1DM.

Accordingly, insulin has been shown to suppress gene expression of apolipoprotein C3 (APOC3) $(12,13)$, an abundant apolipoprotein that increases triglyceride-rich lipoproteins (TRLs) and their remnants in the circulation by blocking their catabolism and clearance (14). Studies in humans have convincingly demonstrated that APOC3 loss-of-function mutations lower TRL levels and offer cardioprotection (15-20). APOC3 was long believed to elevate plasma TRL levels by inhibiting lipoprotein lipase $(14,21)$, but a human antisense oligonucleotide (ASO) therapeutic against APOC3 markedly reduced triglyceride (TG) levels in lipoprotein lipase-deficient humans with severe hypertriglyceridemia (22). Recent studies in mice and humans indicate that APOC3 regulates 
Table 1. Clinical characteristics of subjects in CACTI selected for the present study

\begin{tabular}{lcccc} 
Covariate & T1DM cohort & T1DM CAD cases & HR (95\% CI) & P value \\
No. of subjects & 145 & 47 & & \\
Age (yr) & $37.0(9.0)$ & $43.6(7.9)$ & $1.10(1.06,1.15)$ & $<0.0001$ \\
Sex (female) & $79(54.5 \%)$ & $21(44.7 \%)$ & $0.67(0.35,1.29)$ & 0.23 \\
Duration (yr) & $23.4(8.2)$ & $30.6(7.7)$ & $1.13(1.08,1.19)$ & $<0.0001$ \\
SBP (mmHg) & $118.5(15.6)$ & $126.3(15.0)$ & $1.03(1.02,1.05)$ & 0.0002 \\
DBP (mmHg) & $77.7(9.1)$ & $81.1(9.4)$ & $1.05(1.02,1.08)$ & 0.001 \\
BMI (kg/m²) & $26.3(4.4)$ & $27.3(5.2)$ & $1.05(0.98,1.13)$ & 0.17 \\
Total cholesterol (mg/dL) & $170.5(337)$ & $185.7(30.7)$ & $1.1(1.01,1.02)$ & 0.002 \\
TCs (mg/dL) & $75(58-103)$ & $99(72-132)$ & $2.26(1.40,3.65)$ & 0.0009 \\
HDL-C (mg/dL) & $55.0(16.0)$ & $57.0(19.2)$ & $1.01(0.98,1.03)$ & 0.53 \\
LDL-C (mg/dL) & $96.5(28.2)$ & $105.7(29.7)$ & $1.01(1.00,1.03)$ & 0.03 \\
HbA1c (\%) & $7.7(1.2)$ & $8.4(1.1)$ & $1.42(1.15,1.75)$ & 0.001 \\
Insulin dose per BW (U/kg/d) & $0.65(0.29)$ & $0.62(0.30)$ & $0.74(0.16,3.40)$ & 0.70 \\
CRP ( $\mu$ g /mL) & $1.2(0.9-2.0)$ & $1.4(0.9-2.6)$ & $1.43(0.89,2.28)$ & 0.14 \\
eCFR (mL/min/1.73m²) & $104.5(25.2)$ & $79.6(34.6)$ & $0.97(0.96,0.98)$ & $<0.0001$ \\
Current smoker & $17(11.8 \%)$ & $14(29.8 \%)$ & $3.40(1.54,7.48)$ & 0.002 \\
Medications & & & & \\
ACE inhibitor & $52(35.9 \%)$ & $27(57.4 \%)$ & $2.58(1.34,4.97)$ & 0.005 \\
Angiotensin II receptor blocker & $6(4.1 \%)$ & $4(8.5 \%)$ & $2.35(0.62,8.96)$ & 0.21 \\
Antihypertensive & $60(41.4 \%)$ & $35(74.5 \%)$ & $4.6(2.3,9.5)$ & $<0.0001$ \\
Statin & $24(16.6 \%)$ & $24(51.1 \%)$ & $5.3(2.7,10.5)$ & $<0.0001$ \\
\hline Vat & & &
\end{tabular}

Values are shown as the mean (SD) or as the number of patients (percentage). The total number of subjects included was 181 (11 CAD cases were included in the cohort group consisting of 145 subjects). ${ }^{A}$ Median and IQR. ACE, angiotensin-converting enzyme; eGFR, estimated glomerular filtration rate.

quantified by targeted mass spectrometry (MS). The clinical characteristics of the case and cohort groups are shown in Table 1. Although the cohort's median plasma TG levels were in the normal range, scatterplot analyses showed a positive correlation between serum APOC3 levels and TG levels $\left(r^{2}=0.33 ; P<0.0001\right)$. We observed no significant correlations between APOC3 and LDL-C or HDL-C $(P=0.23$ and $P=$ 0.27 , respectively).

TG levels predicted incident CAD risk with a HR of 1.50 (95\% CI: 1.18-1.90; $P=0.001$; Figure 1). However, serum APOC3 was a stronger predictor, with a HR of 1.84 (95\% CI: $1.40-2.40$; $P<0.0001)$. In 3 different models - model 1, a logistic regression model (Figure 1) adjusted for age, sex, and diabetes duration; model 2, in which model 1 was further adjusted for nonlipid factors (hemoglobin A1c [HbA1c], blood pressure, and current smoking status); and model 3, in which model 1 was further adjusted for lipid factors (LDL-C and HDL-C) - each SD increase in serum APOC3 levels remained associated with a highly significant 1.8 - to 2.0 -fold risk for CAD (Figure 1). Thus, serum APOC3 predicted CAD events in subjects with T1DM and median TGs in the normal range, independently of LDL-C and HDL-C levels. When model 1 was adjusted for log TG levels (model 4 in Figure 1),

multiple steps in TRL metabolism, including inhibition of hepatic uptake of TRLs, slowing the conversion of TRLs to LDL, and inhibition of tissue lipoprotein lipase activity (23-25).

Little is known about the role APOC3 in CVD in patients with T1DM, in part because T1DM is not usually associated with dyslipidemia (26), but is still associated with an increase in CVD risk. We therefore tested the hypothesis that APOC3 is a coronary artery disease (CAD) risk factor in T1DM subjects enrolled in the Coronary Artery Calcification in Type 1 Diabetes (CACTI) study, a prospective evaluation of individuals with T1DM. Our observations indicated that serum levels of APOC3 predict incident CAD in that cohort of patients who had normal plasma TGs. We further demonstrated that APOC3 levels are elevated in mouse models of T1DM and that intensive insulin therapy lowers them. Moreover, we found that lowering APOC3 levels prevented atherosclerosis in a mouse model of T1DM-accelerated atherosclerosis, raising the possibility that APOC 3 could be a therapeutic target in patients with T1DM.

\section{Results}

Serum APOC3 predicts future cardiovascular events in T1DM subjects. We tested the hypothesis that APOC3 levels predict incident CAD in a case-cohort study of 181 T1DM subjects enrolled in CACTI, a prospective study that included several measures, including CAD outcomes (myocardial infarction, coronary artery bypass grafting, angioplasty, or CAD death as reported in the patient's medical record). All subjects were apparently healthy and without known CAD when the samples were collected. Serum APOC3 levels were the HR was $1.45(P=0.11)$. Therefore, the effects of APOC 3 and TGs on CAD in adults with T1DM are not independent.

To further investigate the relationship between APOC3 and TGs, we determined whether adding TGs to a model already adjusted for APOC3 levels, age, sex, and diabetes duration improved the fit of the model, based on the change in the $-2 \log$ likelihood (-2LL). The addition of TGs did not significantly improve the model fit that included APOC3 (change in -2LL $=2.368$, $P=0.12$ ). In contrast, addition of APOC3 to a model that included TGs, age, sex, and diabetes duration significantly improved the model fit (change in $-2 \mathrm{LL}=3.941, P=0.047$ ). This approach indicates that APOC3 is a stronger predictor of CAD than are TG levels in a model adjusted for age, sex, and diabetes duration.

Relative insulin deficiency increases APOC3 levels in mouse models of T1DM. To determine mechanisms through which APOC3 might be increased in T1DM, we used 2 independent mouse models of T1DM: (a) destruction of $\beta$ cells mediated by $C D 8^{+} \mathrm{T}$ cells induced by a virus $(27,28)$ and (b) $\beta$ cell injury induced by streptozotocin (STZ) (29). All the mice were deficient in the LDL receptor $\left(\mathrm{Ldlr}^{\circ}\right.$ ) to mimic a more human-like lipoprotein profile and were fed a lowfat semipurified diet to avoid marked hypercholesterolemia (27).

Plasma APOC3 levels correlated with plasma TG levels in both diabetic mice $\left(r^{2}=0.37, P<0.0001, n=53\right)$ and nondiabetic mice $\left(r^{2}=0.07, P=0.04, n=59\right)$. In the virally induced T1DM model, diabetes resulted in a higher ratio of plasma APOC3 to TG levels. The APOC3/TG ratio was $3.4 \pm 0.3$ (mean \pm SEM, $n=42$ ) in nondiabetic mice versus $4.8 \pm 0.5$ in diabetic mice $(P=0.009 ; n=36)$. Accordingly, at any given TG level within the range of $96^{-460} \mathrm{mg} / \mathrm{dL}$, 


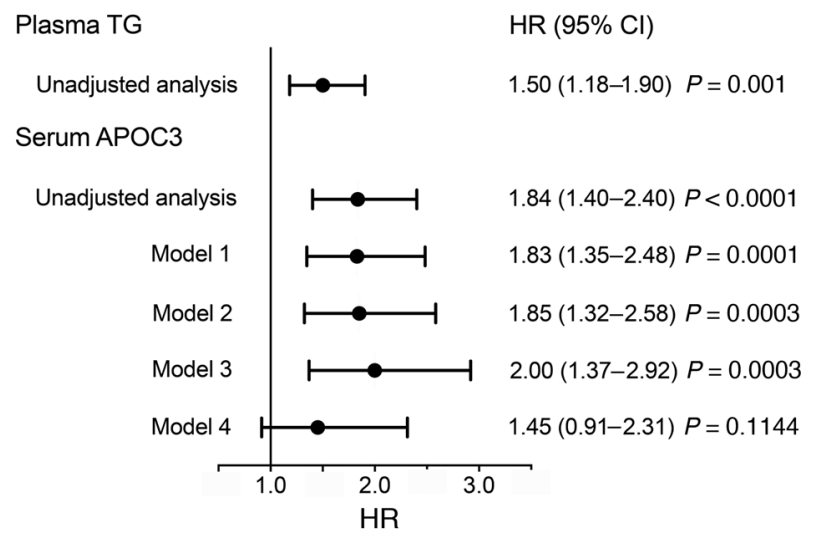

Figure 1. Plasma APOC3 is a strong predictor of CAD events in subjects with T1DM and is independent of traditional CAD risk factors. HRs for CAD events per $1 \mathrm{SD}$ increase in log fasting plasma TGs or log serum APOC3, as calculated by Cox proportional hazards models (47 subjects with events; 181 total subjects). Also shown are $95 \% \mathrm{Cls}$ and $P$ values. A total of 47 participants had a primary endpoint CAD event, defined as a first nonfatal myocardial infarction, coronary revascularization, or death from CAD. Model 1 is a model adjusted for age, sex, and diabetes duration. Model 2 is model 1 further adjusted for nonlipid risk factors: HbA1c, systolic and diastolic blood pressure, and current smoking status. Model 3 is model 1 further adjusted for lipid risk factors: LDL-C and HDL-C. Model 4 is model 1 further adjusted for log fasting TGs.

plasma APOC3 levels were 1.75- to 1.95-fold higher in diabetic mice as compared with levels in their nondiabetic littermates, independent of blood glucose (Figure 2A). Although we did not directly measure APOC 3 production or clearance rates, these observations strongly suggest that APOC3 levels are higher in the mouse model of T1DM than in nondiabetic mice and that the increased APOC3 levels did not simply reflect increased TG levels.

Studies of isolated hepatocytes suggest that hyperglycemia can increase APOC3 production (30). We therefore determined whether dapagliflozin, an inhibitor of sodium glucose transporter 2 (SGLT2), affected APOC3 and lipid levels in mice with STZinduced diabetes. This model was used because STZ-induced diabetic mice retain some endogenous insulin production. Dapagliflozin reduces blood glucose through insulin-independent renal excretion of glucose. It lowered blood glucose levels without significantly affecting plasma TGs, cholesterol, or plasma APOC3 (Figure 2, B-E).

The APOC3 promoter contains an insulin response element (13), and high doses of insulin have been shown to suppress hepatic Apoc3 gene expression in STZ-induced diabetic mice (12). To test whether the elevation in plasma APOC3 we observed in diabetic mice was driven by insulin deficiency, we quantified levels of APOC3, lipids, and glucose in mice with virus-induced diabetes that were either conventionally treated with insulin (sufficient to prevent extensive weight loss and ketonuria) or with an intense insulin regimen to normalize blood glucose. Blood glucose levels were significantly lower in the diabetic mice on intense insulin therapy than in the conventionally treated diabetic mice (Figure 2, F and I, and Supplemental Figure 2, E and F; supplemental material available online with this article; https://doi.org/10.1172/ JCI127308DS1). The mice on intense insulin therapy also had significantly lower plasma APOC3 levels. In contrast, intense insu- lin therapy did not alter plasma cholesterol or TG levels (Figure 2, G-H). Nuclear exclusion of the transcription factor FoxO1 has been proposed to mediate the suppressive effects of insulin on Apoc3 expression (31). To determine whether Apoc3 is regulated by FoxO transcription factors, we measured Apoc 3 mRNA expression in mice with liver-targeted deletions of FoxO1, FoxO3, and FoxO4 (Supplemental Figure 1A), using a previously described model (32). Contrary to experiments based on forced overexpression of FoxO1 (31), we found that hepatocyte deletion of FoxOs did not alter Apoc3 mRNA levels in mice fasted for 4 hours during the dark cycle (Supplemental Figure 1B). Consistent with this observation, injecting adult FoxO triple-floxed mice with an adeno-associated virus (AAV-Tbg-Cre) to induce hepatic FoxO deletion successfully decreased Foxo1 mRNA expression in the liver by $86 \%(P=$ 0.009 ), but did not affect Apoc3 mRNA expression (1.00 \pm 0.14 vs. $1.05 \pm 0.08, P=0.77)$.

To further address whether insulin acts by suppressing the transcription of hepatic Apoc3, we took advantage of a new microplate-based ChIP platform to analyze livers from nondiabetic mice, diabetic mice, and diabetic mice treated with the intense insulin regimen or an acute injection of insulin. We analyzed polymerase II (pol II) binding to the Apoc3 promoter and enhancer and histone H3 modifications associated with active and inactive chromatin. Insulin injections reduced blood glucose levels and induced phosphorylation of Akt and GSK3 $\beta$ in the livers of diabetic mice, as would be expected (Supplemental Figure 2A and B). See complete unedited blots in the supplemental material. Furthermore, insulin resulted in increased pol II binding to the glucokinase (Gck) promoter, a known hepatic target of physiological insulin levels in vivo (ref. 33 and Supplemental Figure 2, C and D). However, insulin did not alter the binding of pol II or modified histones to the Apoc3 promoter or enhancer (Supplemental Figure 2, C and D) after an acute insulin injection (30 $\mathrm{min}$ ) or in the intense insulin treatment experiment, in which a marked reduction of plasma APOC3 was observed (Figure 2I). The suppressive effect of insulin on plasma APOC3 levels had a much slower onset than did the rapid blood glucose-lowering effect of insulin (Supplemental Figure 2, E and F), consistent with the reported half-life of plasma APOC3 (34).

Collectively, these experiments support the conclusion that transcriptional regulation of $A$ poc 3 constitutes a minor pathway for its regulation by diabetes and insulin and that plasma APOC3 levels are elevated in T1DM primarily because of insulin deficiency rather than because blood glucose levels are elevated.

Apoc3 ASO treatment reduces APOC 3 and TRL levels in diabetic mice. To explore the contribution of elevated levels of APOC3 to accelerated atherosclerosis in our virus-induced mouse model of T1DM (27), we used a mouse-specific Apoc3 ASO that has been shown to significantly reduce APOC3 and TG levels in multiple rodent models $(23,35)$. Our 4 groups of mice included (a) nondiabetic control mice treated with a control ASO (cASO); (b) nondiabetic control mice treated with the Apoc3 ASO; (c) diabetic littermate mice treated with the cASO; and (d) diabetic littermate mice treated with the Apoc3 ASO. The mice received the ASO i.p. twice weekly for 12 weeks, starting 2 days after the onset of diabetes (glucose $>250 \mathrm{mg} / \mathrm{dL}$ ).

Plasma glucose levels were similarly elevated in the diabetic mice treated with cASO or Apoc3 ASO (Figure 3A). Apoc3 ASO treatment lowered plasma cholesterol levels in both the nondiabetic 
A

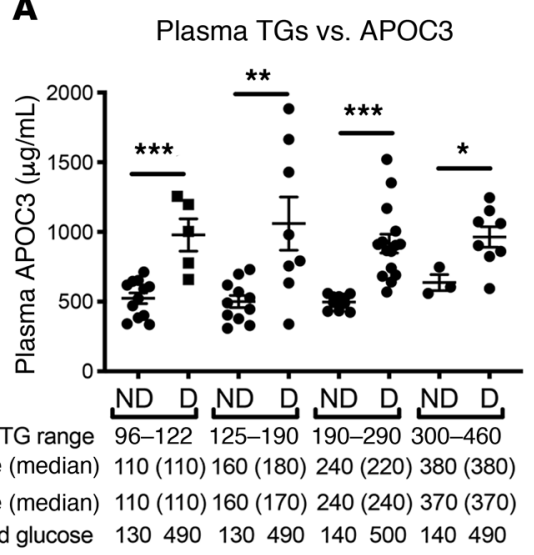

D

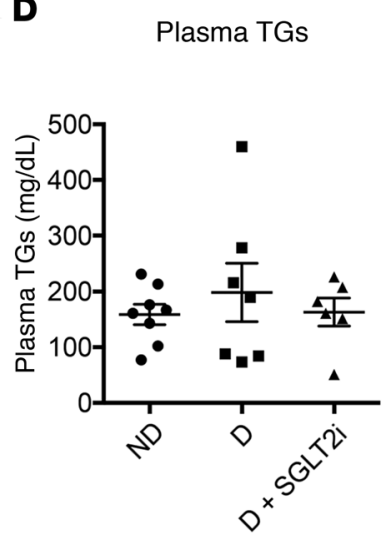

G

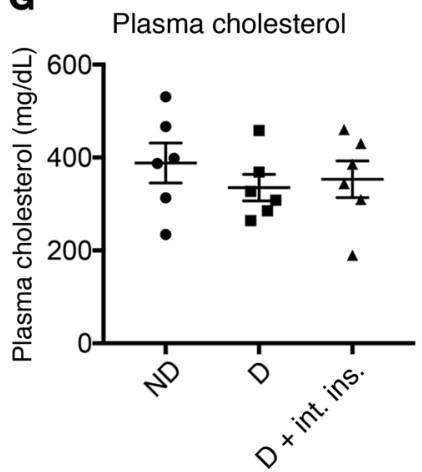

B

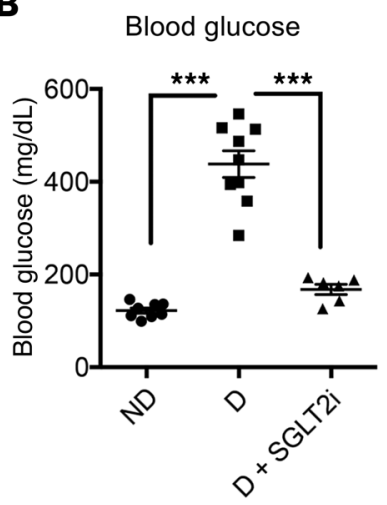

E

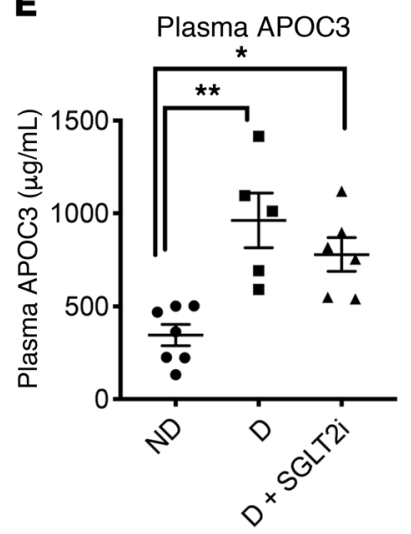

H

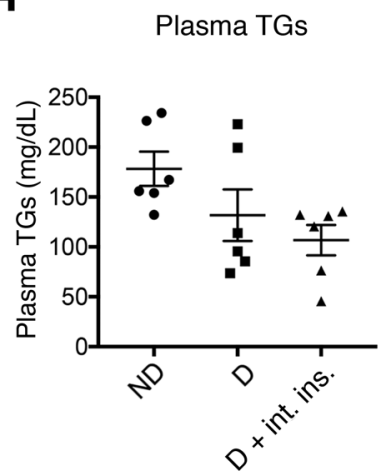

C

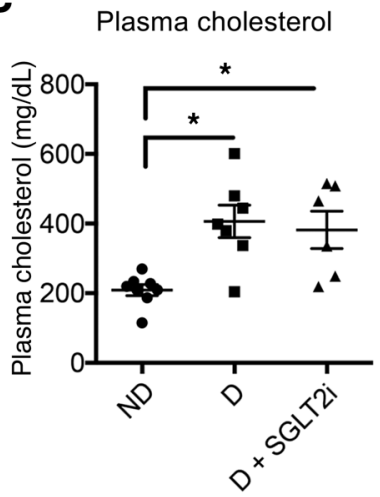

$\mathbf{F}$

Blood glucose

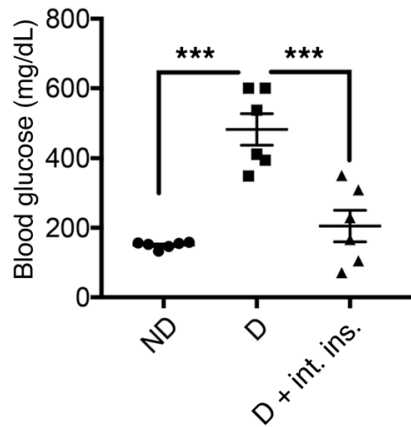

I

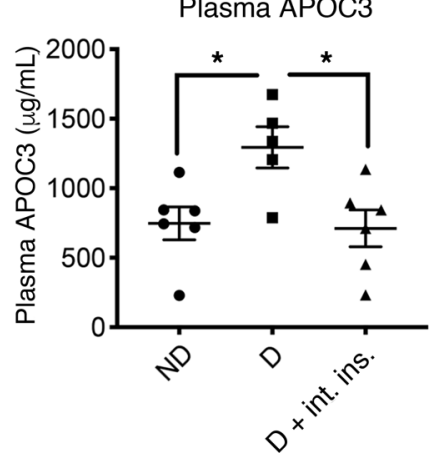

Figure 2. Diabetes increases AРОСЗ levels relative to plasma TG levels through a lack of sufficient insulin. (A) Female $L d l r^{1-} G p^{T g}$ mice were rendered diabetic using LCMV. Saline was used as a control in nondiabetic littermates. At the onset of diabetes, the mice were switched to a low-fat, semipurified diet and maintained on the diet for 4 weeks. Plasma TGs were compared with plasma APOC3 levels measured by ELISA using data from 3 separate cohorts of mice $(n=42-43)$. Ranges and averages of TC levels and blood glucose $(\mathrm{mg} / \mathrm{dL})$ in diabetic and nondiabetic mice are listed below the graph. (B-E) Diabetes was induced by STZ treatment in male $L d I r^{-1-} C p^{T g}$ mice. Following induction of diabetes, half of the diabetic cohort received the SGLT2 inhibitor dapagliflozin in their drinking water for 4 weeks. $n=6-8$. (B) Blood glucose levels at the end of the study. (C) Plasma cholesterol levels. (D) Plasma TGs. (E) Plasma APOC3 levels. Diabetes was induced using LCMV in Ldll $r^{-1-} G p^{T g}$ mice. (F-I) Following development of diabetes, half of the diabetic cohort was subjected to intense insulin therapy with the goal of normalizing blood glucose, whereas the other half was maintained on traditional insulin therapy. $n=$ 5-6. (F) Blood glucose at the end of the study. (C) Plasma cholesterol. (H) Plasma TGs. (I) Plasma APOC3. ND, nondiabetic mice; D, diabetic mice. D + int. ins., diabetes plus intense insulin therapy. ${ }^{*} P<0.05$, ${ }^{* *} P<0.01$, and ${ }^{* * *} P<0.001$, by 2-tailed, unpaired Student's $t$ test (A) or 1 -way ANOVA followed by Tukey's multiple comparisons tests (B-I).

and diabetic mice (Figure 3B), and it also lowered plasma TG levels in the diabetic mice (Figure 3C). At the end of the study, we measured hepatic and intestinal Apoc3 mRNA levels in the 4 mouse groups. We found that the diabetic mice had modestly higher levels of hepatic Apoc3 mRNA than did the nondiabetic controls (Figure
3D), consistent with increased APOC3 production, although plasma levels of APOC 3 were increased to a much greater extent by diabetes. The Apoc3 ASO markedly suppressed hepatic Apoc3 mRNA levels in both nondiabetic and diabetic mice (Figure 3D). We observed no significant effects of diabetes or the Apoc3 ASO on intestinal lev- 
A

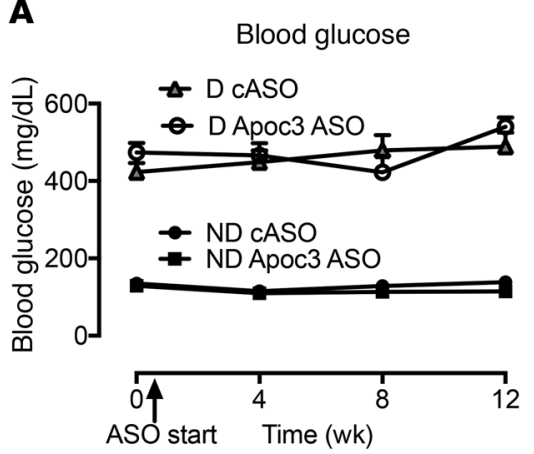

D

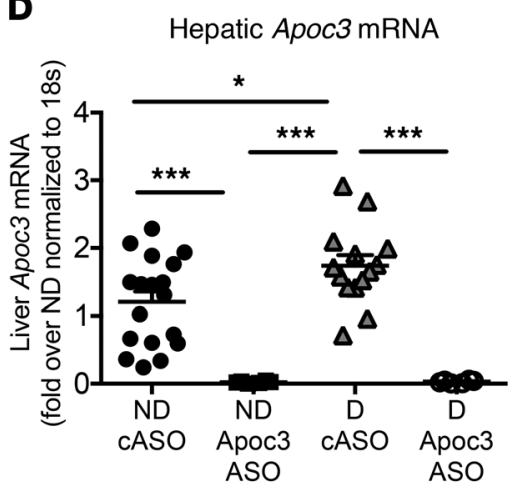

B

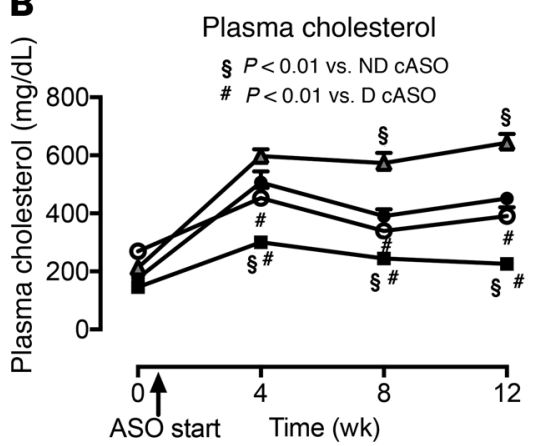

E

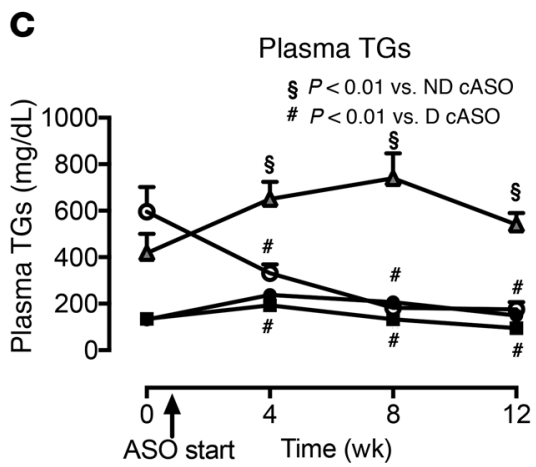

ASO start Time (wk)

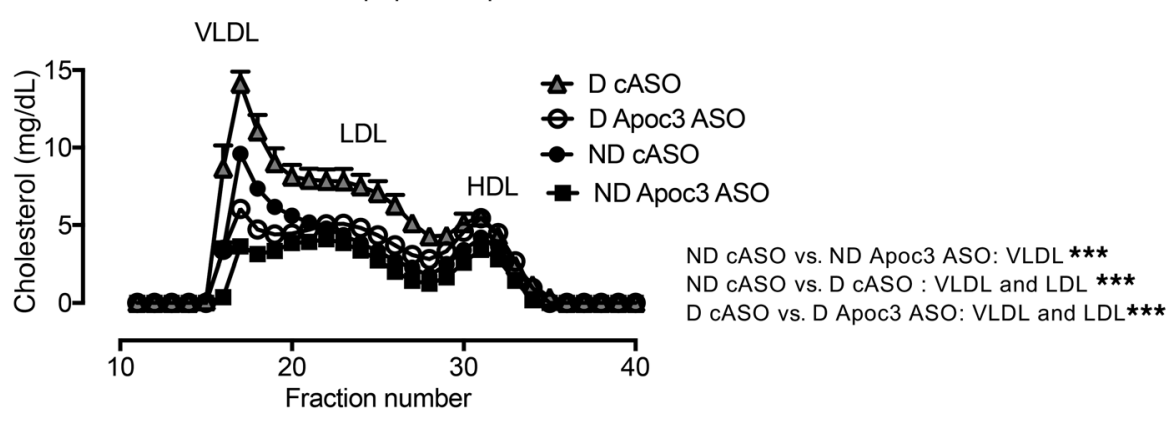

G
H
$\mathbf{F}$

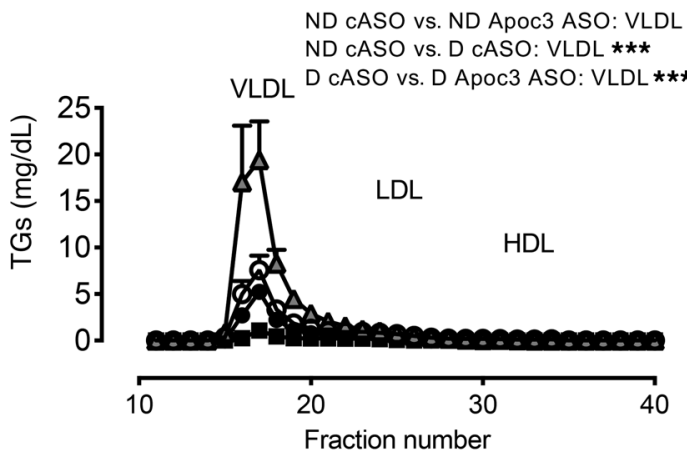

TG lipoprotein
Plasma APOC3

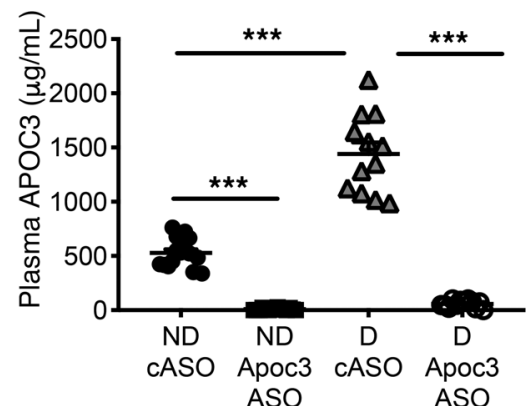

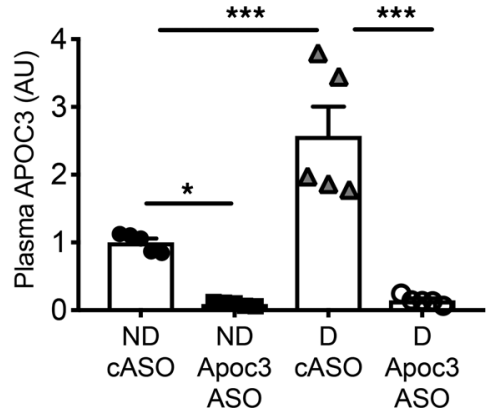

Figure 3. Reducing APOC3 expression with an ASO normalizes TRL levels in diabetic mice. Female $L d l r^{1-}$ G $p^{\text {Tg }}$ mice were rendered diabetic using LCMV. Saline was used as a control in nondiabetic mice. The mice were maintained for 12 weeks. At the onset of diabetes, the animals were switched to a lowfat, semipurified diet. The mice were treated twice weekly with $25 \mathrm{mg} / \mathrm{kg}$ (i.p. injections) Apoc3 ASO or cASO starting 2 days after the onset of diabetes. Doses were adjusted every 2 weeks on the basis of body weight. Animals were bled every 4 weeks for glucose and lipid measurements. (A) Blood glucose levels. (B) Plasma cholesterol levels. Note that time point 0 is before animals were initiated on the low-fat, semipurified diet but after they had developed diabetes. (C) Plasma TGs (A-C; $n=16-20)$. (D) Hepatic mRNA was isolated, and Apoc3 mRNA was measured by real-time PCR ( $n=6-18)$. (E and F) At the end of the study, cholesterol and TG lipoprotein profiles were analyzed in a subset $(n=4)$ of mice. (G) Plasma levels of APOC3, measured by ELISA ( $n=$ 12-16) at the end of the study. (H) The APOC3 ELISA was validated by targeted MS $(n=5-6) .{ }^{*} P<0.05$, and ${ }^{* *} P<0.001$, unless otherwise indicated, by 1-way ANOVA followed by Tukey's multiple comparisons test (D, G, and $\mathbf{H}$ ) or 2-way ANOVA followed by Bonferroni's multiple comparisons test (E and $\mathbf{F}$ ) ${ }^{\#} P<0.01$ compared with D cASO and $\$ P<0.01$ compared with ND CASO, by 2-way ANOVA followed by Bonferroni's multiple comparisons test (B and C).

els of Apoc3 mRNA (Supplemental Figure 3A), as in previous studies that found a less pronounced effect of Apoc3 ASO in the intestine than in the liver (35). These findings are most likely explained by the lower distribution of ASO to the intestine. The Apoc3 ASO did not alter hepatic TG levels or plasma alanine aminotransferase (ALT) levels (Supplemental Figure 3, B and C), indicating that this ASO is well tolerated. These results are consistent with those of previous human and animal studies (35).
Plasma lipoprotein profiles showed that Apoc3 ASO primarily suppressed the increased levels of VLDL cholesterol and VLDL TGs (TRLs), with a smaller effect on IDL/LDL cholesterol (Figure 3, E and F). Analysis of plasma APOC3 levels by ELISA and targeted MS studies confirmed that diabetes was associated with a significant increase in plasma APOC3 levels and that the Apoc3 ASO markedly suppressed plasma APOC3 levels in both nondiabetic and diabetic mice (Figure 3, $\mathrm{G}$ and $\mathrm{H}$, and Supplemental Figure $4 \mathrm{~A}$ ). 
A

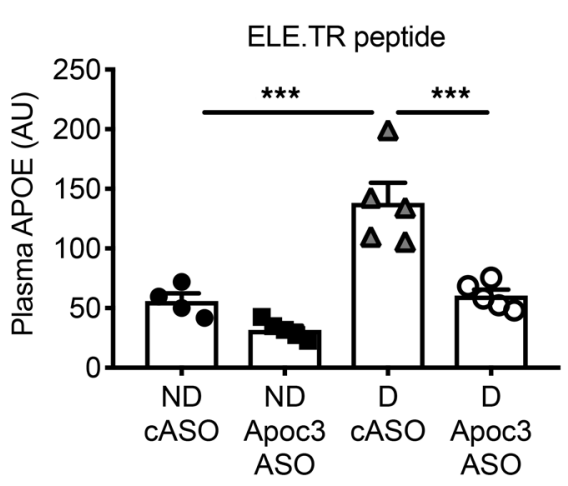

D

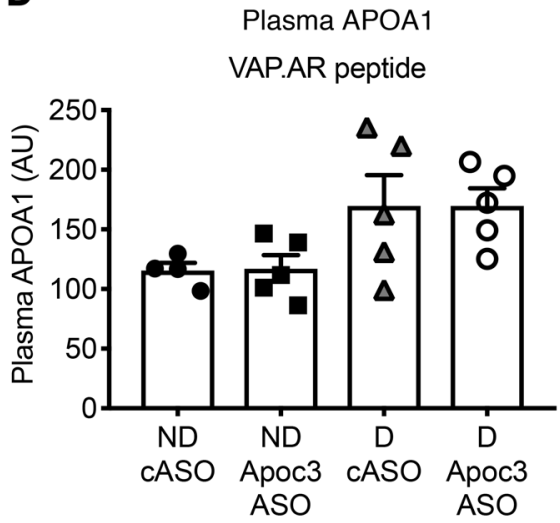

B

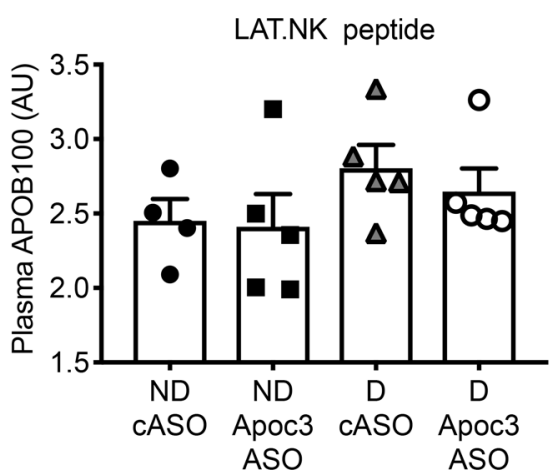

E

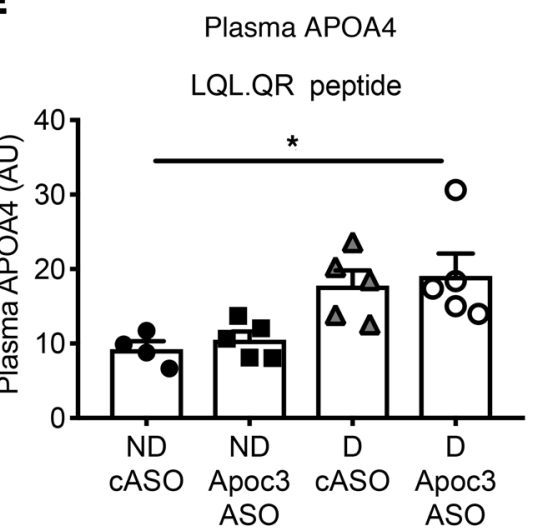

C

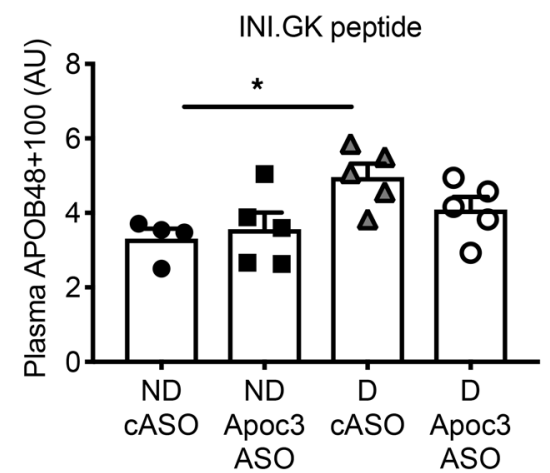

$\mathbf{F}$

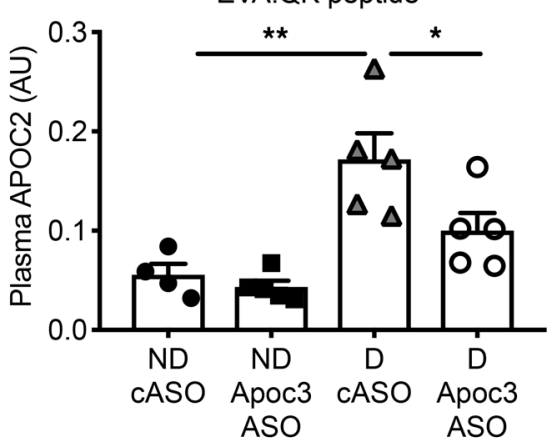

Figure 4. Diabetes results in increased plasma levels of APOE, which are normalized by Apoc3 ASO treatment. Diabetes was induced in female $L d l r^{-1-} G p^{T_{g}}$ mice using LCMV. Saline was used as a control in nondiabetic mice. The mice were maintained for 12 weeks and treated with cASO or Apoc3 ASO. At the end of the study, plasma levels of APOE (A), APOB100 (B), APOB48 plus APOB100 (C), APOA1 (D), APOA4 (E), and APOC2 (F) were measured by targeted MS. The results are expressed as AU. $n=4-5 .{ }^{*} P<0.05,{ }^{*} P<0.01$, and ${ }^{* *} P<0.001$, by 1 -way ANOVA followed by Tukey's multiple comparisons tests.

To get a fuller picture of how diabetes and Apoc3 ASO alter lipid metabolism, we measured several other plasma apolipoproteins by targeted MS. Diabetes increased plasma APOE levels by more than 2-fold over those in nondiabetic mice (Figure $4 \mathrm{~A}$ and Supplemental Figure 4B), an effect that Apoc3 ASO completely prevented. Previous studies have shown that Apoc3 ASO treatment lowers APOE-rich TRLs in nondiabetic human subjects (20), suggesting that diabetes increases the levels of APOE-containing TRLs and their remnants that are normalized by the Apoc3 ASO. Interestingly, the effect of diabetes on APOB plasma levels was much less pronounced: the difference reached statistical significance only when a peptide present in both APOB100 and APOB 48 was quantified (Figure 4, B and C). This suggests that diabetes did not significantly increase the number of APOB100-containing lipoprotein particles and that the Apoc3 ASO did not lower these particles, also consistent with data in nondiabetic human subjects (20). The effects of diabetes on plasma APOA1 (the major protein of HDL) were modest (Figure 4D). We also observed no effect of diabetes or Apoc3 ASO on HDL levels and function that could explain the atheroprotective effects of Apoc3 ASO (see below), despite an almost complete loss of APOC 3 associated with HDL in Apoc3 ASO-treated mice (Supplemental Figure 5). Consistent with an insignificant role for HDL relative to TRLs in mediating the effect of APOC3, HDL-associated APOC3 was a much weaker
CAD risk predictor than was serum APOC 3 in the human CACTI cohort, with a nonsignificant HR of 1.38 (95\% CI: 0.93-2.05, $P=$ 0.11 in the model adjusted for age, diabetes duration, and sex.

Furthermore, in diabetic mice, we observed a modest increase in APOA4 and a marked increase in plasma APOC2, a potent activator of lipoprotein lipase. Antisense inhibition of APOC3 suppressed the increase in APOC2 in the diabetic mice (Figure 4, E and $\mathrm{F}$ ), which was also consistent with human data on nondiabetic individuals (20).

Together, these results support the idea that diabetes primarily affects TRL metabolism, rather than other lipoproteins, and that the impact of Apoc3 ASO on TG levels is mediated in large part by reduced levels of APOE-containing TRLs and their remnants in diabetic mice, which is consistent with other mouse and human studies $(20,24,36)$. In contrast, HDL containing APOA1 and LDL containing APOB100 were not markedly affected by diabetes or Apoc3 ASO treatment in our mouse model. The inability of Apoc3 ASO to increase HDL might be due to the lack of cholesteryl ester transfer protein in rodents (37), as antisense inhibition of APOC3 increases HDL levels concomitantly with TG lowering in humans $(38,39)$.

Apoc3 ASO prevents diabetes-accelerated lesion initiation and macrophage accumulation in artery walls. In order to investigate whether the effects of Apoc3 ASO on lipids translate to reduced atherosclerosis, we quantified atherosclerosis at 2 vascular sites in 
A

En face atherosclerosis (aorta)

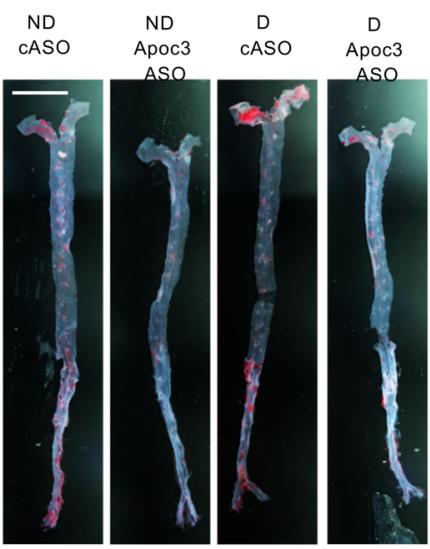

B

BCA lesions

Movat's

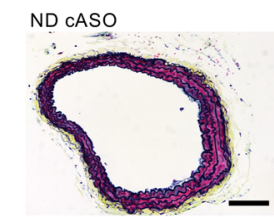

D cASO

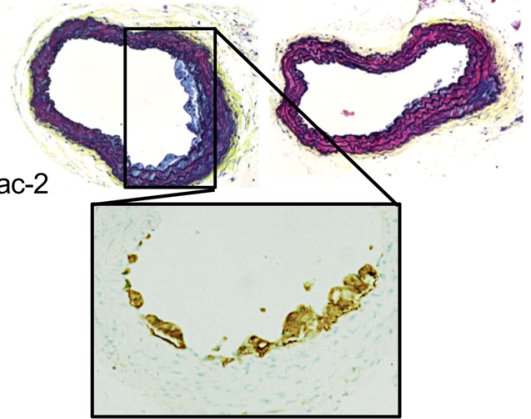

E

En face atherosclerosis

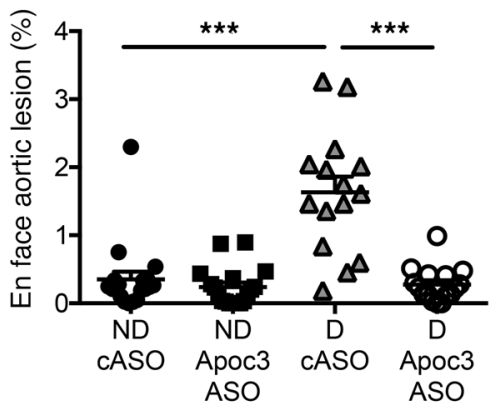

G

BCA APOC3

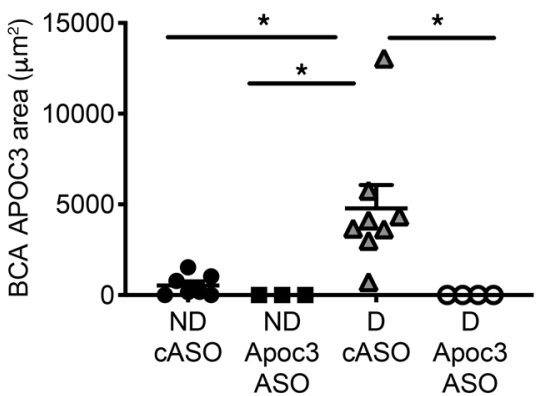

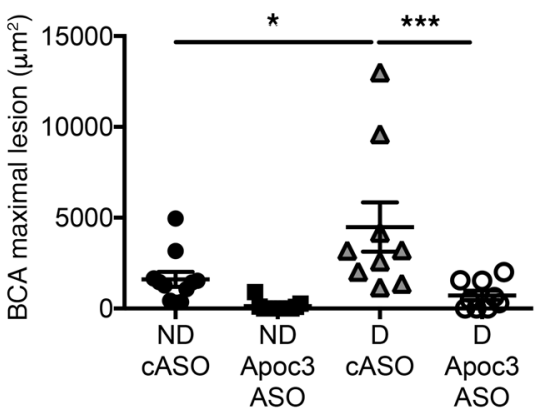

H

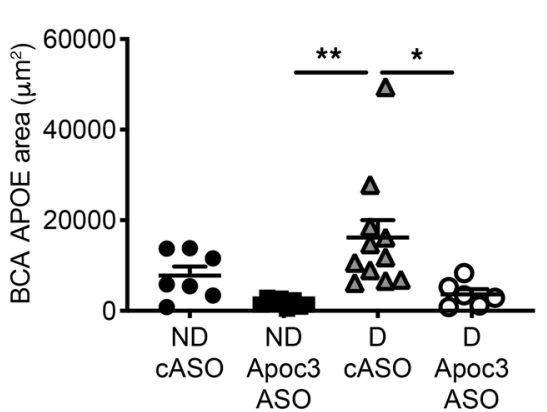

C

BCA APOC3, APOE, and APOB stain

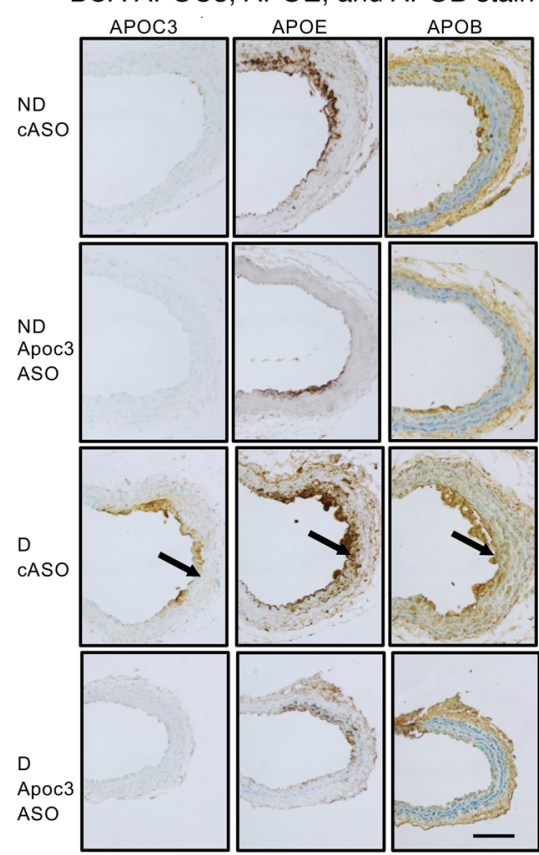

$\mathbf{F}$

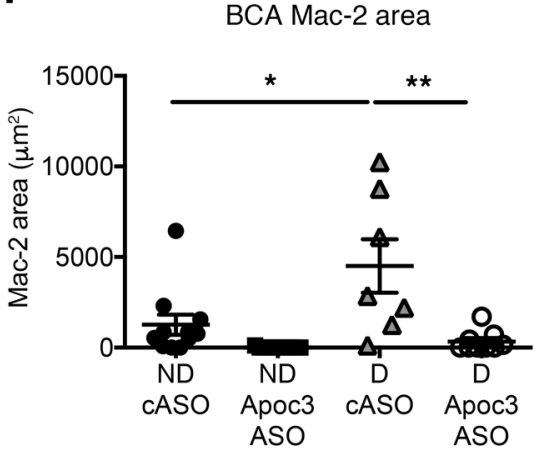

I

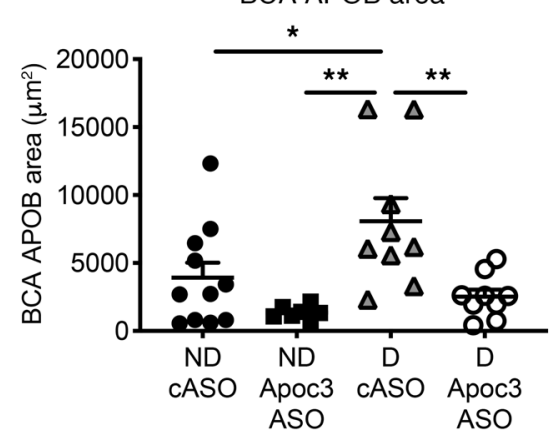

Figure 5. Diabetes-accelerated atherosclerosis is prevented by Apoc3 ASO treatment. Female $L d l r^{-1-} \mathrm{Cp} \mathrm{p}^{T_{g}}$ mice were rendered diabetic using LCMV. Saline was used as a control in nondiabetic mice. The mice were maintained for 12 weeks. At the onset of diabetes, the mice were switched to a low-fat, semipurified diet. Animals were treated twice weekly with $25 \mathrm{mg} / \mathrm{kg}$ (i.p. injections) of Apoc3 ASO or cASO starting 2 days after the onset of diabetes. (A and D) En face aortic atherosclerosis $(n=15-19)$. (B and C) Examples of early lesions in the BCA. Inset in B shows Mac-2 staining at magnified 2-fold from the image above. The internal elastic lamina is indicated by arrows. (E) Quantification of the maximal lesion area in the BCA $(n=9-11)$. (F) Mac-2+ lesion area in BCA cross sections ( $n=7-11)$. (G) Quantification of APOC3 immunoreactivity in the BCA $(n=3-8)$. (H) Quantification of APOE immunoreactivity in the BCA $(n=6-11)$. (I) Quantification of APOB immunoreactivity in the BCA $(n=7-11) * P<0.05,{ }^{* *} P<0.01$, and ${ }^{* * *} P<0.001$, by 1-way ANOVA followed by Tukey's multiple comparisons test. Scale bar: $0.5 \mathrm{~cm}(\mathbf{A})$ and $100 \mu \mathrm{m}$ (B and C). 
A

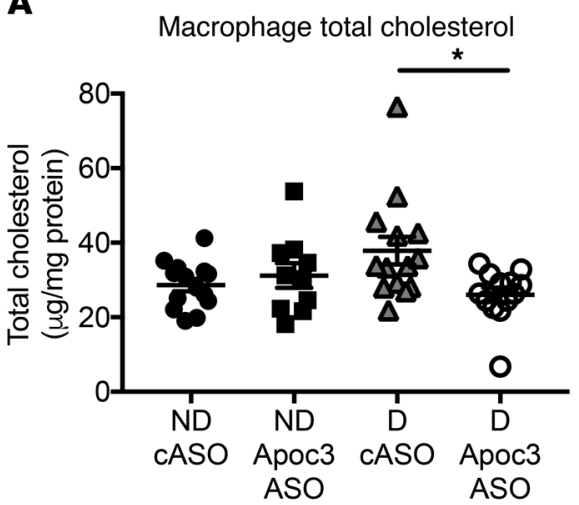

C

Peritoneal fluid cholesterol

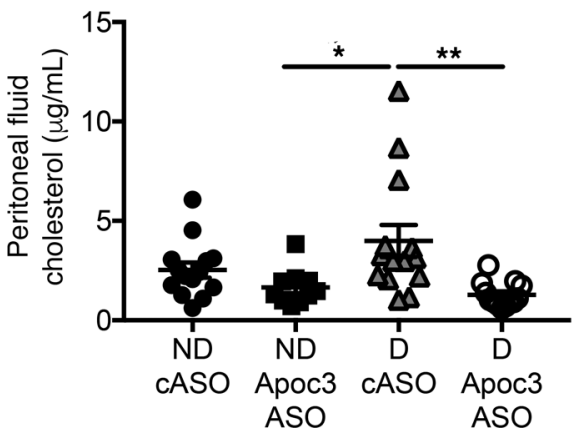

B

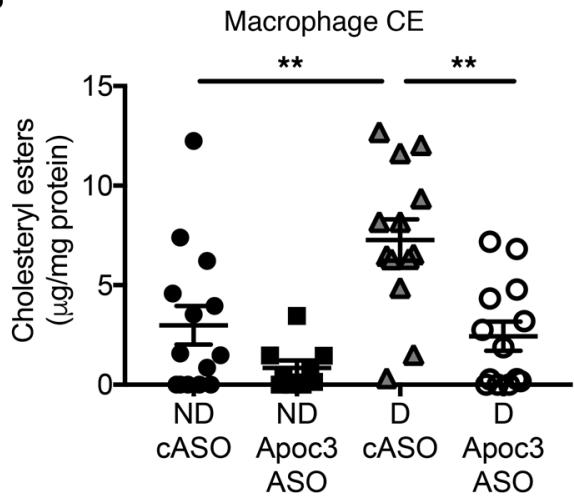

D

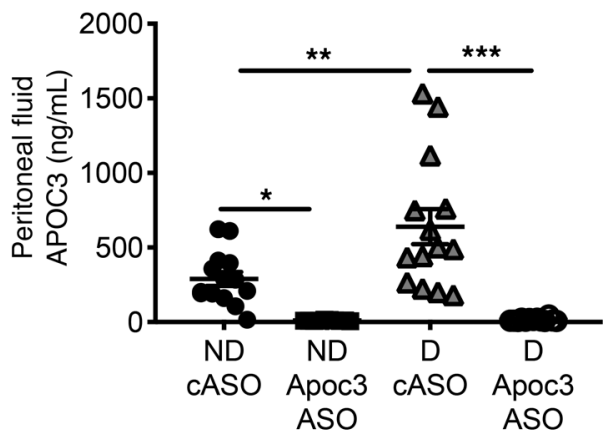

Figure 6. Diabetes increases APOC3 levels in interstitial fluid concomitant with macrophage cholesteryl ester accumulation, both of which are prevented by Apoc 3 ASO. Female $L d l r^{1-} G p^{T g}$ mice were rendered diabetic using LCMV. Saline was used as a control in nondiabetic mice. The mice were maintained for 4 weeks. At the onset of diabetes, the animals were switched to a low-fat, semipurified diet. The mice were treated twice weekly with $25 \mathrm{mg} / \mathrm{kg}$ (i.p. injections) of Apoc3 ASO or CASO starting 2 days after the onset of diabetes. At the end of the study, resident macrophages were isolated by peritoneal lavage, and interstitial peritoneal fluid was collected. (A) Total cholesterol in macrophages. (B) Cholesteryl ester (CE) in macrophages. (C) Cholesterol levels in peritoneal fluid. (D) APOC3 levels in peritoneal fluid. $n=10-14$ (A-D) (2 statistical outliers were removed from $B: 1$ in the ND Apoc3 ASO group and 1 in the D cASO group). ${ }^{*} P<0.05$, ${ }^{* *} P<0.01$, and ${ }^{*}{ }^{*} P<0.001$, by 1-way ANOVA followed by Tukey's multiple comparisons test. all 4 groups of mice (nondiabetic and diabetic mice injected with the cASO and nondiabetic and diabetic littermates injected with the Apoc3 ASO). Lesions in the aorta, from the arch to the iliac artery bifurcation, were analyzed en face and identified as areas positive for the lipophilic stain Sudan IV (Figure 5A). Diabetic mice had larger lesions in the aorta, as demonstrated previously $(27,40)$. Administration of the Apoc3 ASO completely prevented the increase in lesion area in the diabetic mice (Figure $5 \mathrm{~B}$ ). We analyzed the lesions in the brachiocephalic artery (BCA) by serial sectioning of the entire BCA and measuring the cross-sectional lesion area at the site of maximal extent $(27,41)$. Like in the aorta, we observed that diabetes promoted atherosclerosis in the BCA and that this effect was prevented by the Apoc3 ASO (Figure 5, $\mathrm{C}-\mathrm{E})$. The BCA lesions were early fatty streak lesions consisting primarily of macrophages. Diabetes increased macrophage lesion areas (measured by Mac-2 IHC), and Apoc3 ASO prevented this increase (Figure 5F).

Monocytosis can promote atherosclerosis in nondiabetic and diabetic hypercholesterolemic mice (42-44). We therefore quantified the levels of circulating leukocyte populations by flow cytometry to determine whether the Apoc3 ASO suppressed blood monocyte levels. Neither diabetes nor the Apoc3 ASO altered the numbers of WBC, monocytes, Ly6 $\mathrm{C}^{\text {hi }}$ monocytes, or neutrophils (Supplemental Figure 6, A-D). Diabetic mice had elevated platelet levels, as reported in recent studies (45), but administration of the Apoc3 ASO had no significant effect on platelets in our animals (Supplemental Figure 6E). Altered levels of circulating monocytes are therefore unlikely to explain the effect of Apoc3 ASO on lesional macrophage accumulation and atherosclerosis in this mouse model of diabetes.
SAA1 and SAA2 are prominent acute-phase proteins in mice, which have very low levels of $\mathrm{C}$-reactive protein (CRP), the major human acute-phase protein (46). Apoc3 ASO modestly increased SAA levels in our diabetic mice (Supplemental Figure 6F). We also found that peritoneal macrophages from the diabetic mice had increased inflammatory activation, consistent with our previous studies (40). However, Apoc3 ASO did not significantly lower Tnfa or $I l 1 b$ mRNA levels in peritoneal macrophages of the diabetic mice (Supplemental Figure 6, G and H).

Together, these results suggest that the proatherosclerotic effects of APOC3 in diabetic mice reflect increased macrophage accumulation in the artery wall rather than altered levels of circulating myeloid cells or systemic inflammation.

Apoc3 ASO treatment prevents the accumulation of APOC3, $A P O E$, and $A P O B$ in artery walls and macrophage foam cell formation in diabetic mice. Deposition of proatherogenic lipoproteins in the artery wall is a key step in atherogenesis (47). We therefore asked whether arterial accumulation of APOC3-containing lipoproteins is enhanced in diabetic mice. Immunostaining of atherosclerotic lesions, using an antibody against APOC3 (Supplemental Figure 7), revealed larger amounts of APOC3 in the BCA of diabetic mice and complete reversal of this pathology by Apoc3 ASO treatment (Figure 5, C and G). Interestingly, APOC3 was detected in the arterial media, just below the internal elastic lamina and sites of accumulated macrophages, and around macrophages in the intima (Figure 5C). Furthermore, immunohistological staining of adjacent cross sections revealed similar patterns for APOE and APOB (Figure 5, C and G-I). Importantly, diabetes resulted in increased APOB in the artery wall, without significantly affecting 

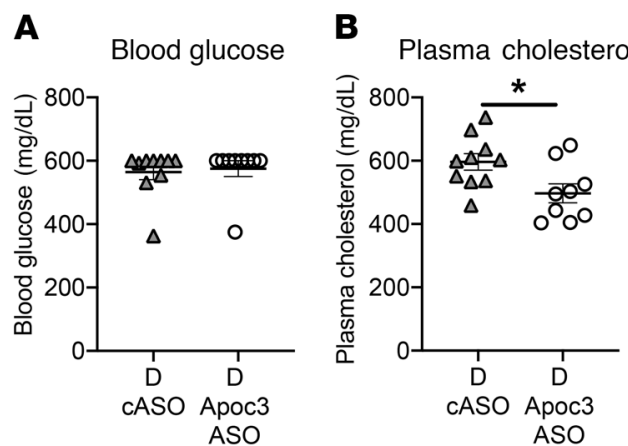

$\mathbf{E}$
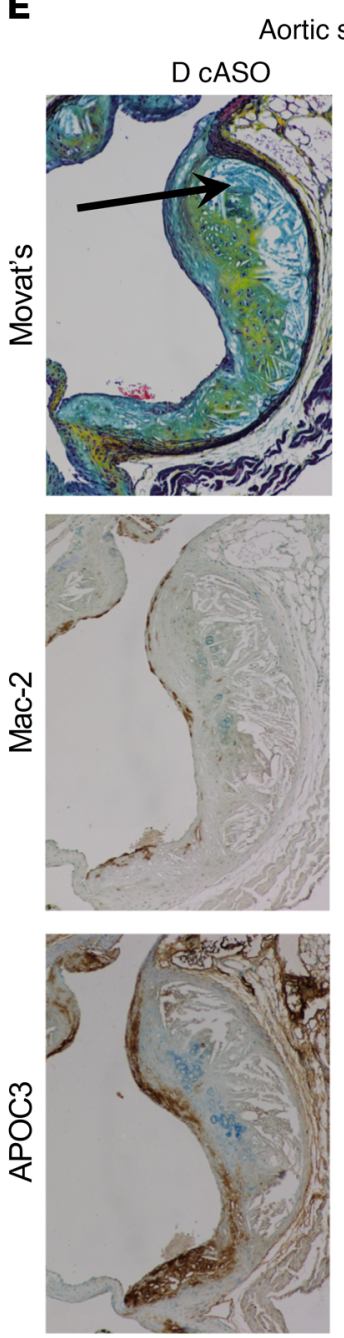

C

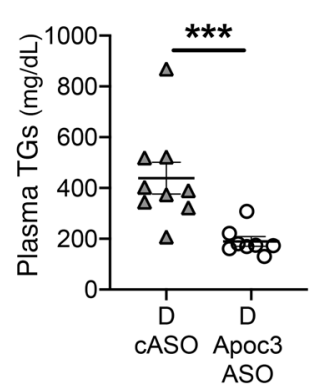

$\mathbf{F}$
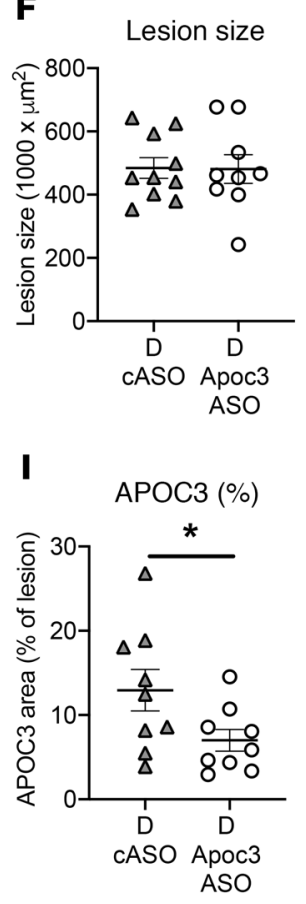

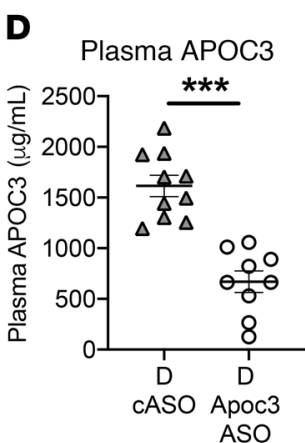

G
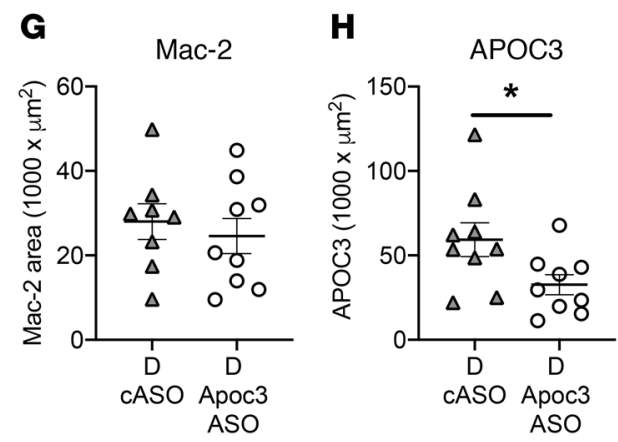

$\mathbf{J}$

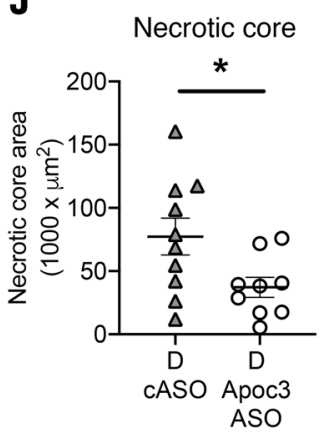

$\mathbf{K}$

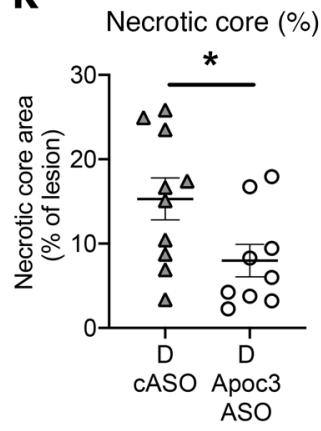

Figure 7. Apoc3 ASO treatment reduces necrotic cores in preexisting lesions in diabetic mice. Female $L d r^{\prime-} G p^{T_{g}}$ mice were fed a high-fat diet containing $1.25 \%$ cholesterol for 12 weeks, switched to chow for 2 weeks, and then injected with LCMV to induce diabetes (D) or with saline (ND). Once diabetic, the mice were maintained for 4 weeks on a low-fat, semipurified diet. The mice were treated twice weekly with $25 \mathrm{mg} / \mathrm{kg}$ (i.p. injections) Apoc3 ASO or cASO starting 2 days after the onset of diabetes. (A) Blood glucose. (B) Plasma cholesterol. (C) Plasma TCs. (D) Plasma APOC3. (E) IHC images of aortic sinus lesions stained with Movat's pentachrome, Mac-2, and APOC3. Arrow indicates the necrotic core. Scale bar: $100 \mu \mathrm{m}$. (F) Quantification of aortic sinus lesion size at $+180 \mu \mathrm{m}$ (largest lesion) after the appearance of all 3 aortic valve leaflets. There were no differences in lesion size at 0 or $+90 \mu \mathrm{m}$. (C) Quantification of aortic lesion Mac-2 staining at $+180 \mu \mathrm{m}$. ( $\mathbf{H}$ and I) Quantification of APOC3 IHC staining at $+180 \mu \mathrm{m}$. (J and K) Quantification of aortic lesion necrotic cores at $+180 \mu \mathrm{m}$. Similar results were observed at $+90 \mu \mathrm{m}$. $n=9-10 .{ }^{*} P<0.05$, and ${ }^{* *} P<0.001$, by 2-tailed, unpaired Student's $t$ test.

plasma APOB100 levels (Figure 4B). These observations suggest that APOC3 promotes the trapping of atherogenic lipoproteins probably remnant particles containing APOC3, APOE and APOB - in the artery wall.

To further investigate the role of foam cell formation in diabetic mice, we harvested peritoneal macrophages and quantified cholesterol and cholesteryl ester levels. Macrophages from the diabetic mice had elevated levels of cholesteryl ester, but not total cholesterol, and cholesteryl ester levels were normalized in macrophages harvested from the diabetic mice we treated with the Apoc3 ASO
(Figure 6, A and B). The accumulation of cholesteryl ester in macrophages from the diabetic mice probably resulted from increased levels of lipoproteins and remnants containing cholesterol and APOC3 in the animals' peritoneal interstitial fluid. These levels decreased markedly after Apoc3 ASO treatment (Figure 6, C and D).

Together, these results suggest that the beneficial effects of Apoc3 ASO on atherosclerosis in the setting of diabetes are due to reduced levels of APOC3-, APOE-, and APOB-containing lipoproteins and remnants within the artery wall and a subsequent reduction in macrophage foam cells. 


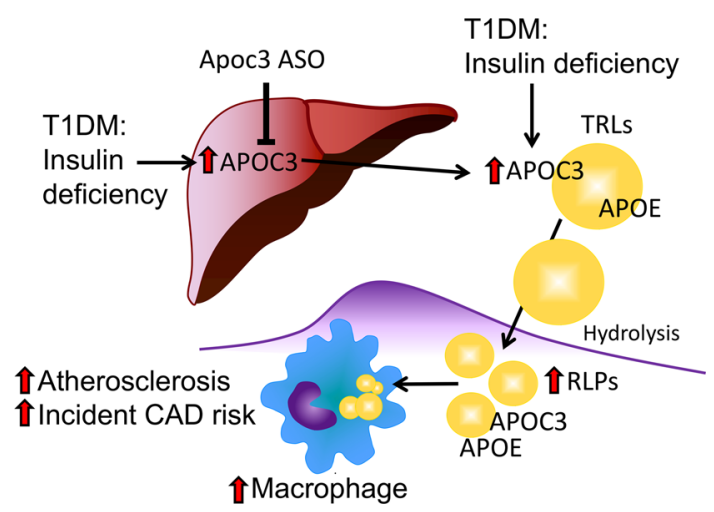

Figure 8. Schematic model. Suboptimally controlled diabetes associated with relative insulin deficiency increases hepatic APOC3 production and, to a larger extent, plasma APOC3 levels. The increased levels of APOC3 prevent clearance of plasma TLRs and their remnant lipoproteins (RLPs). As a result, lipoproteins containing $A P O C 3, A P O E$, and $A P O B$ accumulate in the artery wall and accelerate macrophage accumulation, foam cell formation, atherogenesis, and $C A D$ risk.

Apoc3 ASO treatment reduces necrotic core size in diabetic mice with preexisting lesions. To investigate whether APOC3 accumulation is also increased in more complex lesions under diabetic conditions, we initiated lesion formation by fat-feeding for 12 weeks prior to induction of diabetes (Supplemental Figure 8A). Diabetes was subsequently induced, and the mice were maintained on a low-fat diet for an additional 4 weeks (Supplemental Figure 8A). Diabetes resulted in hyperglycemia and a modest increase in plasma cholesterol levels (Supplemental Figure 8, B and C). Consistent with our other results, plasma TG levels were $108 \pm 11$ $\mathrm{mg} / \mathrm{dL}$ in nondiabetic mice and $402 \pm 87 \mathrm{mg} / \mathrm{dL}$ in diabetic mice (mean \pm SEM; $n=9-10 ; P=0.0056$ ), and plasma APOC3 levels were $454 \pm 37 \mu \mathrm{g} / \mathrm{mL}$ in nondiabetic mice and $1531 \pm 162 \mu \mathrm{g} / \mathrm{mL}$ in diabetic mice (mean \pm SEM; $P=9-10 ; P<0.00001$ ). The short duration of diabetes did not alter the atherosclerotic lesion size of preexisting lesions in the aortic sinus (Supplemental Figure 8, D and $\mathrm{E}$ ). We have previously demonstrated that diabetes results in a more severe lesion phenotype of preexisting lesions (28), and consistently, after only 4 weeks of diabetes, the mice had increased necrotic core sizes (Supplemental Figure 8, D and G). This lesion phenotype was associated with a dramatic increase in lesional APOC3 accumulation (Supplemental Figure 8, D and $\mathrm{H}$ ).

Next, to test whether Apoc3 ASO treatment could prevent the progression of advanced lesions characterized by increased necrotic cores in diabetic mice, we induced preestablished lesions by fat feeding for 12 weeks, followed by induction of diabetes and treatment with Apoc3 ASO or cASO. As shown in Figure 7, A-D, Apoc3 ASO treatment did not alter blood glucose levels, but modestly suppressed plasma cholesterol and markedly suppressed TG and plasma APOC3 levels, similar to the results of the lesion initiation study (Figure 3). Apoc3 ASO treatment did not reduce total lesion size or macrophage accumulation as assessed by Mac-2 staining in preexisting lesions (Figures 7, E-G). Strikingly, Apoc3 ASO treatment significantly reduced lesional APOC3 accumulation and necrotic core size (Figure 7, E and $\mathrm{H}-\mathrm{K})$, suggesting that inhibition of APOC3 prevents the pro- gression of lesions into more advanced lesions characterized by larger necrotic cores.

\section{Discussion}

Our observations indicate that serum levels of APOC3 predict incident CAD risk in a cohort of T1DM patients with median TG levels in the normal range $(<150 \mathrm{mg} / \mathrm{dL})$ independently of LDL-C, HDL-C, and multiple other nonlipid risk factors, including diabetes duration and HbA1c. When we adjusted the model for TGs, APOC3 was no longer a significant predictor of CAD, probably reflecting the strong links between APOC3 and TGs. However, adding APOC3 to an analysis that adjusted for TGs, age, sex, and diabetes duration significantly improved the fit of the model to the data $(P$ value $=0.047)$. In contrast, adding TGs to an analysis that adjusted for APOC3 did not improve the fit of the model. Taken together, these observations support the proposal that elevated levels of APOC3 are a risk factor for CAD in patients with T1DM and that, although APOC3 and TGs are not independent predictors, APOC 3 is a stronger predictor of CAD than are TGs in this cohort of subjects with T1DM.

Importantly, our mouse studies demonstrated that diabetes increases plasma levels of APOC3. Moreover, lowering APOC3 levels with an ASO against APOC3 markedly reduced atherosclerosis in diabetic mice, likely by reducing levels of atherogenic lipoproteins containing APOC3, APOE, and APOB. These findings raise the possibility that APOC 3 could be a therapeutic target in humans with T1DM.

What could explain a heightened importance of APOC3 as a CAD risk factor in T1DM? Previous mouse studies have failed to demonstrate a beneficial effect of APOC3 deficiency on atherosclerosis in nondiabetic mice fed an atherogenic diet (48). Our findings that plasma APOC3 levels were approximately 2-fold higher in diabetic mice than in control mice over a wide range of TG levels and that hepatic Apoc3 expression was likewise increased in diabetic mice shed light on this question. These results suggest that plasma APOC3 levels are elevated not simply because of increased retention of TRLs in plasma, but through a mechanism tied to the T1DM condition. Hepatic Apoc3 transcription has been shown to be increased by elevated glucose levels through the transcription factors HNF- $4 \alpha$ and ChREBP in cultured cells $(12,30)$. However, hyperglycemia was not responsible for the elevated plasma APOC3 levels in our diabetic mouse models, as we used an SGLT2 inhibitor to normalize blood glucose levels and found no significant reduction in APOC3 levels. Instead, we found that intensive insulin therapy normalized plasma levels of APOC3.

Forced overexpression of FoxO1 has been demonstrated to induce hepatic Apoc3 expression (31), and FoxO1 is believed to mediate the effects of insulin on Apoc3 expression. However, our data using mice deficient in all FoxOs in the liver suggest that neither FoxO1, FoxO3, or FoxO4 is required for hepatic Apoc3 expression. Furthermore, PIXUL-ChIP (49) analyses of Apoc3 transcriptional regulation in diabetic and insulin-treated diabetic mice failed to reveal significant transcriptional regulation of Apoc3. Together, these results indicate that the marked increase in plasma APOC3 in diabetic mice and the normalization by intense insulin treatment are probably mediated by posttranscriptional effects and by altered kinetics of plasma APOC3. One possibility 
is that insulin reduces plasma APOC3 levels by increasing hepatic uptake of remnant lipoproteins through translocation of LDLRrelated protein 1 (50), a protein involved in APOC3 clearance (23). The exact mechanism of insulin suppression of APOC3 in the setting of diabetes will be an interesting area for future studies. We have previously shown that intensive insulin therapy prevents atherosclerosis in a virus-induced mouse model of T1DM-accelerated atherosclerosis (27), a finding consistent with a role for relative insulin deficiency in promoting atherosclerosis in T1DM.

Volanesorsen, a human APOC3 antisense drug, effectively lowers plasma TG levels in humans with markedly elevated TG levels due to lipoprotein lipase deficiency, without lowering LDL cholesterol (22). This antisense therapeutic agent also reduces TG levels in subjects with T2DM and in subjects with hypertriglyceridemia and dyslipidemia $(38,39)$. A pilot study suggested that volanesorsen is also effective in individuals with normal TG levels (35). Our findings suggest that elevated levels of APOC3 at least partly explains the increased risk of CAD in T1DM patients with normal TGs and that an APOC3 ASO might significantly reduce APOC3 levels - and perhaps CVD risk - in these patients. In future studies, it will be important to investigate the relationship between glycemic control, insulin sensitivity, and APOC3 levels in both T1DM and T2DM subjects.

What is the mechanism by which increased APOC3 accelerates atherosclerosis in diabetes? We found elevated levels of APOE in proteolytic digests of plasma proteins from our diabetic mice, strongly suggesting that elevated levels of APOC3 increase levels of atherogenic TRL remnant lipoproteins rich in APOE and APOB. Consistent with this idea, we found that the elevated APOE levels in diabetic mice were normalized by treatment with Apoc3 ASO. Previous mechanistic studies demonstrated that APOC3 blocks clearance by the liver of lipoproteins containing APOE, perhaps by interfering with APOE-mediated hepatic uptake of those lipoproteins (51-53). In contrast, we found no evidence that APOC3 promotes systemic inflammation, nor did we find any evidence of increased circulating levels of monocytes in diabetic mice.

Instead, elevated APOC3 likely acts in diabetes by promoting the accumulation of atherogenic lipoproteins containing APOC3, APOE, and APOB in the artery wall. An attractive hypothesis is that lipoprotein remnants containing APOC3, APOE, and APOB are trapped in the artery wall, which would in turn increase monocyte recruitment and the accumulation of macrophage foam cells in lesions. Our demonstration that APOC3 accumulates in macrophage-rich regions of both early and advanced atherosclerotic lesions and in medial areas below lesions is consistent with this proposal (Figure 8). It is possible that APOC3 enhances the binding of APOB-containing lipoproteins to extracellular matrix (54, 55), or that the increased presence of atherogenic lipoprotein remnants in the artery wall is responsible for accelerated atherosclerosis. More research is needed to address whether the atherogenic effects are due to direct effects of APOC3 or to atherogenic effects of a particular APOC3-containing lipoprotein particle. We also found that APOC3 levels increased in the interstitial peritoneal fluid of diabetic mice and that peritoneal macrophages of diabetic mice had increased levels of cholesteryl ester, the biochemical hallmark of macrophage foam cells. Our observations are consistent with the "response-to-retention" hypothesis of Williams and Tabas (47), which suggests that retention of cholesterol-rich lipoproteins in the artery wall provokes a cascade of responses that initiate and advance atherosclerosis. Our data further suggest that increased arterial retention of APOC3-, APOE-, and APOBcontaining lipoproteins is responsible for the increased atherosclerosis initiation and progression in the setting of diabetes.

Collectively, our observations raise the possibility that APOC3 is an important risk factor for atherosclerotic CVD in T1DM patients with normal TG levels and that lowering of APOC3 levels might reduce the excess risk for CVD observed in those individuals.

\section{Methods}

CACTI study participants and clinical measurements. The CACTI study has been described previously (56). Briefly, 1416 participants (652 patients with T1DM and 764 nondiabetic subjects), 19-56 years of age, have been followed prospectively at the University of Colorado for a variety of endpoints, including calcium artery score and CVD events. None of the subjects had a known history of CVD, including angina, coronary artery bypass grafting, and coronary angioplasty, and all patients with T1DM had been diagnosed with T1DM before age 30 or had positive autoantibodies or a clinical course consistent with T1DM and had been treated with insulin within 1 year of diagnosis. The mean duration of diabetes was 23 years at enrollment, and the minimum duration was 4 years. After an overnight fast, blood was collected and centrifuged, and separated plasma and serum were stored at $4^{\circ} \mathrm{C}$ until the assay. Total cholesterol and TG levels were measured using standard enzymatic methods. HDL-C was separated using dextran sulfate, and LDL-C was calculated using the Friedewald formula. HPLC was used to measure HbA1c (Bio-Rad variant).

We designed a case cohort study to test the prespecified hypothesis that elevated serum APOC3 predicts CAD events in subjects with T1DM. CAD events were defined as myocardial infarction, coronary artery bypass grafting, angioplasty, or CAD death, as reported in the patient's medical record. Events were ascertained through annual surveillance questionnaires sent to all study participants, through medical history questionnaires assessed at all in-person visits, through reports of events made by study participants, family members, and physicians, and through death records. Death record searches were conducted through the Colorado Department of Public Health Vital Records Department and the National Death Index (NDI) database. Medical records were requested for all events that occurred at a medical facility. Adjudication of events was performed by blinded analysis of those records by a morbidity and mortality committee composed of 3 physicians. The subcohort consisted of 145 subjects with T1DM randomly selected from the overall CACTI cohort, which included 11 of the CAD cases. The cases included 47 subjects with CAD events that occurred after baseline blood collection. All T1DM subjects enrolled in CACTI who had an adjudicated CAD event were included in the analysis. All the subjects were free of known CAD at the time of blood collection. One subject of the 181 was on TG-lowering medication (gemfibrozil). The clinical characteristics of the 2 groups are listed in Table 1. The risk for a CAD event was modeled using weighted Cox proportional hazards linear regression. Strata were analyzed for each CAD event, with subcohort subjects serving as controls for each stratum CAD event that occurred while the subcohort member remained at risk for a CAD event (e.g., prior to experiencing a CAD event and pri- 
or to censoring). The log likelihood ratio test was used to determine a change in model fit when TGs and APOC 3 were added to the adjusted models. The strength of the association was evaluated using HRs.

Mouse models of T1DM-accelerated atherosclerosis. The $\mathrm{T}$ cellinduced LDL receptor-deficient ( $\left(d l^{-/-}\right) G p^{T g}$ mouse model of T1DM-accelerated atherosclerosis has been described previously (27). These mice express the lymphocytic choriomeningitis virus (LCMV) glycoprotein (GP) under control of the insulin promoter, allowing reliable induction of diabetes due to $\mathrm{CD} 8^{+} \mathrm{T}$ cell-mediated $\beta$ cell destruction following LCMV injection. In the current study, 10to 14-week-old female $L d l r^{--} G p^{T g}$ mice on a C57BL/6J background were injected with LCMV. Nondiabetic littermates were injected with saline. At the onset of diabetes (defined as blood glucose levels $>250$ $\mathrm{mg} / \mathrm{dL}$ ) 10 days after LCMV injection, the nondiabetic and diabetic mice were fed a low-fat, semipurified diet without added cholesterol, as previously described (27). The diabetic mice received s.c. insulin pellets (LinShin Canada Inc.) to provide baseline insulin and were treated as needed with insulin glargine (Lantus; Sanofi) to prevent weight loss and ketonuria. The second mouse model of diabetesaccelerated atherosclerosis used in this study was the STZ-treated $\mathrm{Ldlr}^{-/-}$model, also described previously (29). Diabetes was induced in male $\mathrm{Ldlr}^{-}-\mathrm{Gp}^{T g}$ mice by i.p. injection of STZ (mixed anomers no. S0130, $50 \mathrm{mg} / \mathrm{kg}$; MilliporeSigma) dissolved in freshly made $0.1 \mathrm{M}$ sodium citrate buffer ( $\mathrm{pH} 4.5)$ for 5 consecutive days. Control mice were injected with vehicle citrate buffer. Diabetes was defined in these mice as described above, and the animals were treated with insulin glargine as needed to prevent significant weight loss and ketonuria. A subset of the STZ-treated diabetic mice were treated with the SGLT2 inhibitor dapagliflozin at $25 \mathrm{mg} / \mathrm{kg} / \mathrm{day}$ in the drinking water to reduce blood glucose levels, based on the method described by Nagareddy et al. (42). Water intake was measured initially to verify dosing. To test the effect of an intense insulin regimen on plasma APOC3 levels, we injected female $\mathrm{Ldlr}^{-/} G p^{T g}$ mice with LCMV to induce diabetes. At the onset of diabetes, the mice were allocated to either the conventional treatment (see above) or to an intense insulin treatment group. For intense treatment, the mice received insulin via pellets as well as 3 daily injections (7-10 hours apart) of insulin glargine, with the goal of keeping the mice normoglycemic $(<200 \mathrm{mg} / \mathrm{dL})$ for 9 days. The mice were euthanized in the morning, 10 hours after their last insulin injection. Another group of mice were rendered diabetic with LCMV and maintained for 10 days, and a subset of mice received an acute injection of insulin $30 \mathrm{~min}$ utes prior to euthanasia (0.2-0.3 U/ mouse). Prior to the acute injection, blood glucose and plasma APOC3 levels were similar between the 2 diabetic groups and were elevated compared with the levels in the nondiabetic mice. For both the SGLT2 inhibitor administration and intense insulin experiment, mice were randomized to treatment groups according to similarities of initial blood glucose levels.

To study atherosclerotic lesion initiation, mice were rendered diabetic and maintained for 12 weeks on a low-fat, semipurified diet (27, 40). For studies of preexisting lesions, mice were first fed a high-fat, semipurified diet $(40 \%$ calories from fat, $1.25 \%$ cholesterol) $(27,28)$ for 12 weeks and were then switched to normal mouse chow (to reduce plasma cholesterol) and then injected with LCMV to induce diabetes. Once diabetes had developed, the mice were fed the low-fat, semipurified diet and maintained on this regimen for an additional 4 weeks (Supplemental Figure 8A).
At the end of the subsets of experiments, we isolated macrophages from the peritoneal cavity using ice-cold PBS plus 5 mM EDTA. The macrophages were allowed to adhere for 1 hour before they were harvested for either RNA isolation (Nucleospin RNA Plus, Macherey-Nagel) or measurements of total cholesterol, free cholesterol, and cholesteryl esters (Amplex Red Cholesterol Assay; Thermo Fisher Scientific).

Apoc3 ASO treatment. The mouse-specific Apoc3 ASO (5'-CCAGCTTTATTAGGGACAGC-3') and a cASO (CCTTCCCTGAAGGTTCCTCC) were 20-nt 5-10-5 2'-O-methoxyethyl (MOE) gapmers, with underlining indicating 2 'MOE modified bases, produced by Ionis Pharmaceuticals (35). The ASOs $(25 \mathrm{mg} / \mathrm{kg}$ ) were injected i.p. twice weekly, starting 2 days after the onset of diabetes. ASO doses were adjusted every 2 weeks on the basis of body weight. The ASOs were well tolerated in the mice.

Analysis of glucose and lipid metabolism. Blood glucose was measured in saphenous vein blood by stick tests (OneTouch Ultra; LifeScan). As the glucometer does not go beyond $600 \mathrm{mg} / \mathrm{dL}$, values above that were set to $600 \mathrm{mg} / \mathrm{dL}$. Plasma cholesterol levels were determined using the Cholesterol E Kit (Wako Diagnostics), and plasma and liver TGs were determined with a colorimetric kit from MilliporeSigma. Plasma lipoprotein profiles were analyzed by fast protein liquid chromatography (FPLC), as described previously (27). Cholesterol and TGs were measured in each fraction. HDL functional assays and analysis of HDL particle concentrations were performed as described in the Supplemental Methods. To test for hepatotoxicity, we measured plasma levels of the liver enzyme ALT with a kit from MilliporeSigma (MAK052-1KT). Plasma APOC3 levels were measured using an APOC3 ELISA from Abcam (ab217777) and verified using targeted MS (see below).

Quantification of atherosclerosis. The aorta was dissected after in situ perfusion with PBS and then fixed in 10\% phosphate-buffered formalin (MilliporeSigma). Aortae were opened longitudinally (from the heart to the iliac bifurcation), and an investigator blinded to the treatment groups quantified the extent of atherosclerosis en face after Sudan IV staining, as previously described $(27,40)$. BCAs and aortic sinuses were cross-sectioned and stained with the Movat's pentachrome stain to visualize lesion morphology. The aortic sinus was analyzed at 3 separate sites beginning at the appearance of all 3 aortic valve leaflets (57). Lesional macrophages were visualized by Mac-2 IHC, using a monoclonal rat anti-mouse Mac-2 antibody ( $1 \mu \mathrm{g} / \mathrm{mL}$ CL8942AP, Cedarlane) (28). APOC3 IHC was performed with a rabbit polyclonal APOC3 generated by Ionis Pharmaceuticals (1:1000 dilution). APOB IHC was performed using a biotinylated goat anti-APOB antibody (BAF3556, R\&D Systems; at 1:50), and APOE IHC was carried out using a rabbit monoclonal antibody (ab183597, Abcam; at 1:2000). Negative isotype controls (or serum controls) of the same concentrations or dilutions were used as controls (Supplemental Figure 7).

Flow cytometric analysis of blood leukocytes. Mice were bled from the retro-orbital plexus under isoflurane sedation after 12 weeks of diabetes. EDTA was used as the anticoagulant. Total leukocytes were measured using an automated cell counter for mouse blood samples (HEMAVET, Drew Scientific). For flow cytometric analysis, erythrocytes were lysed with an ammonium-chloride-potassium buffer and discarded, and leukocytes were stained using a fixable viability dye, CD45, CD115, and GR1 (clones 30-F11, AFS98, and RB6-8C5, respectively; eBioscience, Thermo Fisher Scientific). Monocytes were identified as CD115 cells, and neutrophils were identified as CD115- GR $1^{\text {hi }}$ 
cells. All analyses were performed with live, single, $\mathrm{CD} 45^{+}$cells. The monocytes were further divided into $\mathrm{GR}^{\mathrm{hi}}\left(\mathrm{Ly}_{6} \mathrm{C}^{\text {hi }}\right)$ and GR1 ${ }^{\text {lo }}\left(\mathrm{Ly}_{6} \mathrm{C}^{\mathrm{lo}}\right)$ subpopulations. The cells were analyzed on a BD FACS RUO Flow Cytometer (BD Biosciences). Flow cytometric analysis was normalized to total WBC counts and expressed as cells $/ \mathrm{mL}$ blood, with the assumption that all WBC were CD $45^{+}$.

Real-time PCR. Gene expression in liver, intestine, and resident peritoneal macrophages was quantified by real-time PCR. RNA isolation and the real-time PCR protocol were performed as described previously (40). Briefly, RNA was isolated using QIAGEN RNeasy or Macherey-Nagel Nucleospin RNA kits according to the manufacturers' protocols. RNA samples were treated with DNase1 $(1 \mu \mathrm{g} / \mathrm{sample}$; Thermo Fisher Scientific) to remove traces of DNA. Real-time PCR was performed using the SYBR Green 1 detection method (Thermo Fisher Scientific). Ct values were normalized to Rn18s and presented as the fold change versus the control. Primer sequences are available in the supplemental materials.

Analysis by liquid chromatography-electrospray ionization tandem MS of serum, plasma, and HDL-associated proteins. Liquid chromatography-electrospray ionization targeted tandem MS (LC-ESI-MS/MS) analysis was used to determine selected proteins in human serum, mouse plasma, and human and mouse HDL, as described below and in the Supplemental Methods.

Digestion of human serum. In a digestion plate, $5 \mu \mathrm{L}$ serum was diluted in $245 \mu \mathrm{L}$ of $25 \mathrm{mM} \mathrm{NH}_{4} \mathrm{HCO}_{3}$. An aliquot of $10 \mu \mathrm{L}$ diluted serum (equivalent to $0.2 \mu \mathrm{L}$ serum and $\sim 14 \mu$ g protein) was placed into a new digestion plate. Following the addition of freshly prepared methionine ( $5 \mathrm{mM}$ final concentration) in $20 \%$ acetonitrile and $100 \mathrm{mM}$ $\mathrm{NH}_{4} \mathrm{HCO}_{3}$, serum proteins $(\sim 14 \mu \mathrm{g})$ were reduced with dithiothreitol and then alkylated with iodoacetamide. After adding $0.2 \mu \mathrm{g}$ isotopelabeled $\left[{ }^{15} \mathrm{~N}\right]$ APOA1 (as the internal standard), the serum was incubated at $37^{\circ} \mathrm{C}$ with 50:1 (w/w, proteins/enzyme) of sequencing-grade modified trypsin (Promega) for 4 hours. A second aliquot of trypsin (1:25 final, w/w, enzyme/proteins) was added, and the samples were incubated overnight at $37^{\circ} \mathrm{C}$. Digestion was halted by acidifying the reaction mixture $(\mathrm{pH} 2-3)$ with trifluoroacetic acid, and the samples were dried and stored at $-80^{\circ} \mathrm{C}$ until MS analysis.

Mouse plasma digestion. Plasma digestion was performed as follows: $3 \mu \mathrm{L}$ plasma was diluted 20 times in $100 \mathrm{mM} \mathrm{NH}_{4} \mathrm{HCO}_{3}$, and $10 \mu \mathrm{L}$ diluted plasma was diluted to $50 \mu \mathrm{L}$ with $0.625 \%$ sodium deoxycholate (SDC) in $100 \mathrm{mM} \mathrm{NH}_{4} \mathrm{HCO}_{3}$ and spiked with $0.05 \mu \mathrm{g}$ ${ }^{15} \mathrm{~N}$-APOA1, followed by reduction with DTT $\left(30\right.$ minutes at $60^{\circ} \mathrm{C}$ ) and then alkylation with iodoacetamide (IAA) (45 minutes at room temperature in the dark). Excess IAA was quenched with additional DTT, and the sample was digested with $3 \mu$ g sequencing-grade tryp$\sin$ (Promega; 1:10 w/w to plasma proteins). SDC was precipitated by addition of $5 \mu \mathrm{L} 20 \%$ trifluoroacetic acid, and the precipitates were spun down. Sixty microliters was transferred into a PCR plate and frozen at $-20^{\circ} \mathrm{C}$ until analysis.

Quantification of human serum APOC3 by parallel reaction monitoring with ${ }^{15} \mathrm{~N}$-labeled APOA1. To quantitatively measure the relative levels of APOC3 in serum, we used targeted proteomics with parallel reaction monitoring (PRM), which quantifies multiple proteins as accurately as selected reaction monitoring (SRM) and biochemical approaches (58). A nanoACQUITY UPLC (Waters) was used for the separation, with a linear gradient of $0.1 \%$ formic acid in water (solvent $\mathrm{A}$ ) and $0.1 \%$ formic acid in acetonitrile (solvent $\mathrm{B}$ ). After the dried peptide digests were reconstituted in $0.1 \%$ formic acid, serum peptide digests (equivalent to $0.2 \mu$ g protein) were desalted on a C18 trap column $(0.1 \times 40 \mathrm{~mm})$ packed in-house with Magic C-18 reversephase resin ( $5 \mu \mathrm{m}$; 100 Å; Michrom Bioresources) for 8 minutes at 2.5 $\mu \mathrm{L} / \mathrm{min}$ in $99 \%$ solvent $\mathrm{A}$, and then separated using a C-18 analytical column $(0.1 \times 150 \mathrm{~mm})$ packed in-house with Magic C-18 reversephase resin ( $5 \mu \mathrm{m}$; $100 \AA$; Michrom Bioresources) with uncoated SilicaTip Emitters (20 $\mu \mathrm{m}$ ID, $10-\mu \mathrm{m}$ tip ID; New Objective). The column was kept at $50^{\circ} \mathrm{C}$ and the peptides were eluted from the trap column onto the analytical column at a flow rate of $0.6 \mu \mathrm{L} / \mathrm{min}$ and separated using the following multistep gradient: $1 \%-7 \%$ solvent $\mathrm{B}$ in 1 minute; $7 \%-25 \%$ solvent B in 27 minutes; and $25 \%-35 \%$ solvent $\mathrm{B}$ in 8 minutes. The column was subsequently washed for 3 minutes at $80 \%$ solvent B and reequilibrated at $99 \%$ solvent A for 12 minutes. Peptide digests were analyzed with an ultra-high-resolution accurate mass Orbitrap Fusion Lumos Tribrid Mass Spectrometer (Thermo Fisher Scientific). The mass spectrometer was operated in dataindependent acquisition PRM mode. A full-scan MS survey (from $\mathrm{m} / \mathrm{z}$ 350-1800) was acquired in the Orbitrap at a resolution of 120,000 at $400 \mathrm{~m} / z$. A targeted list of precursor ions with charge state 2 or 3 were isolated and fragmented using higher-energy collisional dissociation fragmentation with $30 \%$ collision energy with a stepped collision energy of $5 \%$, and detected at a mass resolution of 15,000 at $400 \mathrm{~m} / z$ with a maximum injection time of $20 \mathrm{~ms}$ and an automated gain control target of 10,000.

Targeted proteomics data analysis of human serum. Two peptides of APOC3 were selected for quantitative analysis by PRM (59). The MS data were analyzed with the open-source program Skyline (60) to determine the total peak area of all the transitions detected for each peptide. The relative levels of each peptide were calculated by the ratio of the total peak area of all the transitions for each peptide to the total peak area of all the transitions for $\left[{ }^{15} \mathrm{~N}\right]$ THLAPYSDELR (the internal standard peptide derived from $\left.\left[{ }^{15} \mathrm{~N}\right] \mathrm{APOA} 1\right)(59)$. To calculate the relative levels of the peptide between the cohort and CAD groups, we set the average ratio of the peptide in control subjects to an arbitrary unit of 1 . To obtain the relative levels of APOC3, the relative levels of the 2 peptides from the protein were averaged.

Mouse plasma targeted proteomics analysis. The abundance of the selected mouse plasma proteins was quantified by MS using targeted proteomics. Recombinant mouse APOC3 was used as a standard for mouse APOC3 plasma analysis. Tryptic digests were desalted on a C18 trapping column (Reprosil-Pur 120 C18-AQ, 5 $\mu \mathrm{m}, 0.1 \times 40 \mathrm{~mm}$, Dr. Maisch HPLC GmbH) (trapping flow rate: $4 \mu \mathrm{L} / \mathrm{min}$ ) and separated on a capillary analytical column (Reprosil-Pur 120 C18-AQ, $5 \mu \mathrm{m}, 250 \times 0.075 \mathrm{~mm}$, Dr. Maisch HPLC $\mathrm{GmbH}$ ) with a 30 -minute linear gradient of acetonitrile and $0.1 \%$ formic acid (7\%-35\%) in $0.1 \%$ formic acid in water at a flow rate of $0.4 \mu \mathrm{L} / \mathrm{min}$ using a nanoACQUITY UPLC (Waters). Analysis by MS was done on either a Thermo TSQ Vantage (Thermo Fisher Scientific) triple-quadrupole mass spectrometer in SRM mode or a Thermo Orbitrap Fusion in PRM mode. For the SRM analysis, the instrument was operated with a 10-ms dwell time, and the selected transitions for the peptide of interest were monitored with collision energies optimized to maximize the signal. For the PRM analysis, the instrument was operated in the targeted mode with 2 Thompson (Th) precursor selection in the quadrupole analyzer and in MS/MS mode with higher-energy collisional dissociation 
activation (30\% normalized collision energy), 30,000 resolution, a maximum injection time of $54 \mathrm{~ms}$, and an automated gain control target of $5 \times 10^{4}$.

Statistics. Statistical analyses of the mouse data were performed using GraphPad Prism 7.0 (GraphPad Software). A 2-tailed, unpaired Student's $t$ test was used to compare differences between 2 groups. A 1- or 2-way ANOVA was used followed by Tukey's or Bonferroni's post hoc test, respectively, to compare 3 or more groups. D'Agostino-Pearson omnibus normality tests were performed to determine whether the data were normally distributed. Statistical outliers were identified by the ROUT $(\mathrm{Q}=1 \%)$ method. Statistical outliers were removed in Figure $6 \mathrm{D}$ and Supplemental Figure 6B, as indicated in the figure legends. Data that were not normally distributed were analyzed by KruskalWallis tests followed by Dunn's multiple comparisons test (for multiple groups) or a Mann-Whitney $U$ test (for 2 groups). A $P$ value of less than 0.05 was considered statistically significant.

Study approval. All human subjects provided informed consent, and the study was approved by the Colorado Combined Institutional Review Board (protocol 97-661). The animal experiments were performed using a protocol approved by the University of Washington IACUC (protocol 3154-01) or the Columbia University IACUC (protocol AAAQ4433).

Note added in proof. A very recent study, published after submission of the final version of this manuscript, demonstrates that total serum APOC3 predicts CVD events in a different cohort of patients with T1DM (the Epidemiology of Diabetes Interventions and Complications cohort), although the association did not reach statistical significance after adjusting for multiple testing of the many biomarkers analyzed (61).

\section{Author contributions}

KEB and JWH designed and directed the study. JEK, TV, BS, JWH, and KEB analyzed data and wrote the manuscript. MJG, RMC, BS, $\mathrm{RAH}$, and TV contributed to the study design and manuscript preparation. JEK, FK, SB, BS, MSA, CRM, DM, KB, and TV performed experiments. JKSB, JEH, and GLK collected data in the CACTI study and analyzed the CACTI cohort CAD data. All authors reviewed the manuscript and provided final approval for submission.

\section{Acknowledgments}

This study was supported by NIH grants R01HL126028, DP3DK108209, R01HL127694, and P01HL092969 (KEB). Part of the study was supported by the Diabetes Research Center at the University of Washington (P3ODK017047) and the American Diabetes Association (1-16-IBS-153, to JEK).

Address correspondence to: Karin Bornfeldt, University of Washington School of Medicine, Department of Medicine, Division of Metabolism, Endocrinology and Nutrition, 750 Republican Street, Seattle, Washington 98109, USA. Phone: 206. 543.1681; Email: bornf@ uw.edu. Or to: Jay Heinecke, University of Washington School of Medicine, Department of Medicine, Division of Metabolism, Endocrinology and Nutrition, 850 Republican Street, Seattle, Washington 98109, USA. Phone: 206.221.5366; Email: heinecke@uw.edu.
1. Emerging Risk Factors Collaboration, et al. Diabetes mellitus, fasting blood glucose concentration, and risk of vascular disease: a collaborative meta-analysis of 102 prospective studies. Lancet. 2010;375(9733):2215-2222.

2. Rawshani A, et al. Mortality and Cardiovascular Disease in Type 1 and Type 2 Diabetes. N Engl J Med. 2017;376(15):1407-1418.

3. Laakso M, Kuusisto J. Insulin resistance and hyperglycaemia in cardiovascular disease development. Nat Rev Endocrinol. 2014;10(5):293-302.

4. Lachin JM, Orchard TJ, Nathan DM, DCCT/ EDIC Research Group. Update on cardiovascular outcomes at 30 years of the diabetes control and complications trial/epidemiology of diabetes interventions and complications study. Diabetes Care. 2014;37(1):39-43.

5. Nathan DM, McGee P, Steffes MW, Lachin JM, DCCT/EDIC Research Group. Relationship of glycated albumin to blood glucose and HbA1c values and to retinopathy, nephropathy, and cardiovascular outcomes in the DCCT/EDIC study. Diabetes. 2014;63(1):282-290.

6. Writing Group for the DCCT/EDIC Research Group, et al. Association between 7 years of intensive treatment of type 1 diabetes and longterm mortality. JAMA. 2015;313(1):45-53.

7. Bebu I, et al. The relationship of blood glucose with cardiovascular disease is mediated over time by traditional risk factors in type 1 diabetes: the DCCT/EDIC study. Diabetologia. 2017;60(10):2084-2091.

8. Cooper ME, El-Osta A, Allen TJ, Watson AMD, Thomas MC, Jandeleit-Dahm KAM. Metabolic
Karma-The Atherogenic Legacy of Diabetes: The 2017 Edwin Bierman Award Lecture. Diabetes. 2018;67(5):785-790.

9. Shah MS, Brownlee M. Molecular and cellular mechanisms of cardiovascular disorders in diabetes. Circ Res. 2016;118(11):1808-1829.

10. Bornfeldt KE. Does elevated glucose promote atherosclerosis? Pros and cons. Circ Res. 2016;119(2):190-193.

11. Chait A, Bornfeldt KE. Diabetes and atherosclerosis: is there a role for hyperglycemia? J Lipid Res. 2009;50 Suppl:S335-S339.

12. Chen M, Breslow JL, Li W, Leff T. Transcriptional regulation of the apoC-III gene by insulin in diabetic mice: correlation with changes in plasma triglyceride levels. J Lipid Res. 1994;35(11):1918-1924.

13. Li WW, Dammerman MM, Smith JD, Metzger S, Breslow JL, Leff T. Common genetic variation in the promoter of the human apo CIII gene abolishes regulation by insulin and may contribute to hypertriglyceridemia. J Clin Invest. 1995;96(6):2601-2605.

14. Taskinen MR, Borén J. Why is apolipoprotein CIII emerging as a novel therapeutic target to reduce the burden of cardiovascular disease? Curr Atheroscler Rep. 2016;18(10):59.

15. Pollin TI, et al. A null mutation in human APOC3 confers a favorable plasma lipid profile and apparent cardioprotection. Science. 2008;322(5908):1702-1705.

16. Saleheen D, et al. Human knockouts and phenotypic analysis in a cohort with a high rate of consanguinity. Nature. 2017;544(7649):235-239.

17. TG HDL Working Group of the Exome Sequenc- ing Project, National Heart, Lung, Blood Institute, et al. Loss-of-function mutations in APOC3, triglycerides, and coronary disease. $N$ Engl J Med. 2014;371(1):22-31.

18. Jørgensen AB, Frikke-Schmidt R, Nordestgaard BG, Tybjærg-Hansen A. Loss-of-function mutations in APOC3 and risk of ischemic vascular disease. N Engl JMed. 2014;371(1):32-41.

19. Khetarpal SA, et al. A human APOC3 missense variant and monoclonal antibody accelerate apoCIII clearance and lower triglyceride-rich lipoprotein levels. Nat Med. 2017;23(9):1086-1094.

20. Pechlaner R, et al. Very-low-density lipoprotein-associated apolipoproteins predict cardiovascular events and are lowered by inhibition of APOC-III. J Am Coll Cardiol. 2017;69(7):789-800.

21. Larsson M, et al. Apolipoprotein C-III inhibits triglyceride hydrolysis by GPIHBP1-bound LPL. JLipid Res. 2017;58(9):1893-1902.

22. Gaudet D, et al. Targeting APOC3 in the familial chylomicronemia syndrome. $\mathrm{NEnglJMed}$. 2014;371(23):2200-2206.

23. Gordts PL, et al. ApoC-III inhibits clearance of triglyceride-rich lipoproteins through LDL family receptors. J Clin Invest. 2016;126(8):2855-2866.

24. Reyes-Soffer G, et al. Effects of APOC3 heterozygous deficiency on plasma lipid and lipoprotein metabolism. Arterioscler Thromb Vasc Biol. 2019;39(1):63-72.

25. Ramms B, et al. ApoC-III ASO promotes tissue LPL activity in absence of ApoE-mediated TRL clearance. J Lipid Res. 2019;null(8):jlr.M093740. 
26. Ginsberg HN. Lipoprotein physiology in nondiabetic and diabetic states. Relationship to atherogenesis. Diabetes Care. 1991;14(9):839-855.

27. Renard CB, et al. Diabetes and diabetes-associated lipid abnormalities have distinct effects on initiation and progression of atherosclerotic lesions. JClin Invest. 2004;114(5):659-668.

28. Johansson F, et al. Type 1 diabetes promotes disruption of advanced atherosclerotic lesions in LDL receptor-deficient mice. Proc Natl Acad Sci US A. 2008;105(6):2082-2087.

29. Wall VZ, et al. Inflammatory stimuli induce acyl-CoA thioesterase 7 and remodeling of phospholipids containing unsaturated long $(\geq \mathrm{C} 20$ )-acyl chains in macrophages. J Lipid Res. 2017;58(6):1174-1185.

30. Caron S, et al. Transcriptional activation of apolipoprotein CIII expression by glucose may contribute to diabetic dyslipidemia. Arterioscler Thromb Vasc Biol. 2011;31(3):513-519.

31. Altomonte J, et al. Foxo1 mediates insulin action on apoC-III and triglyceride metabolism. J Clin Invest. 2004;114(10):1493-1503.

32. Lee SX, et al. FoxO transcription factors are required for hepatic HDL cholesterol clearance. JClin Invest. 2018;128(4):1615-1626.

33. Batista TM, et al. Multi-dimensional transcriptional remodeling by physiological insulin in vivo. Cell Rep. 2019;26(12):3429-3443.e3.

34. Huff MW, Fidge NH, Nestel PJ, Billington T, Watson B. Metabolism of C-apolipoproteins: kinetics of C-II, C-III1 and C-III2, and VLDLapolipoprotein B in normal and hyperlipoproteinemic subjects. J Lipid Res. 1981;22(8):1235-1246.

35. Graham MJ, et al. Antisense oligonucleotide inhibition of apolipoprotein C-III reduces plasma triglycerides in rodents, nonhuman primates, and humans. Circ Res. 2013;112(11):1479-1490.

36. Sacks FM. The crucial roles of apolipoproteins $\mathrm{E}$ and C-III in apoB lipoprotein metabolism in normolipidemia and hypertriglyceridemia. Curr Opin Lipidol. 2015;26(1):56-63.

37. Bell TA, Graham MJ, Baker BF, Crooke RM. Therapeutic inhibition of apoC-III for the treatment of hypertriglyceridemia. JClin Lipidol. 2015;10(2):191-203.

38. Digenio A, et al. Antisense-mediated lowering of plasma apolipoprotein C-III by volanesorsen improves dyslipidemia and insulin sensitivity in Type 2 diabetes. Diabetes Care. 2016;39(8):1408-1415.
39. Gaudet D, et al. Antisense inhibition of apolipoprotein C-III in patients with hypertriglyceridemia. N Engl J Med. 2015;373(5):438-447.

40. Kanter JE, et al. Diabetes promotes an inflammatory macrophage phenotype and atherosclerosis through acyl-CoA synthetase 1. Proc Natl Acad Sci U S A. 2012;109(12):E715-E724.

41. MacDougall ED, et al. Aggressive very lowdensity lipoprotein (VLDL) and LDL lowering by gene transfer of the VLDL receptor combined with a low-fat diet regimen induces regression and reduces macrophage content in advanced atherosclerotic lesions in LDL receptor-deficient mice. Am J Pathol. 2006;168(6):2064-2073.

42. Nagareddy PR, et al. Hyperglycemia promotes myelopoiesis and impairs the resolution of atherosclerosis. Cell Metab. 2013;17(5):695-708.

43. Soehnlein O, Swirski FK. Hypercholesterolemia links hematopoiesis with atherosclerosis. Trends Endocrinol Metab. 2013;24(3):129-136.

44. Tall AR, Yvan-Charvet L, Westerterp M, Murphy AJ. Cholesterol efflux: a novel regulator of myelopoiesis and atherogenesis. Arterioscler Thromb Vasc Biol. 2012;32(11):2547-2552.

45. Kraakman MJ, et al. Neutrophil-derived S100 calcium-binding proteins $\mathrm{A} 8 / \mathrm{A} 9$ promote reticulated thrombocytosis and atherogenesis in diabetes. J Clin Invest. 2017;127(6):2133-2147.

46. Le PT, Muller MT, Mortensen RF. Acute phase reactants of mice. I. Isolation of serum amyloid P-component (SAP) and its induction by a monokine. JImmunol. 1982;129(2):665-672.

47. Williams KJ, Tabas I. The response-to-retention hypothesis of early atherogenesis. Arterioscler Thromb Vasc Biol. 1995;15(5):551-561.

48. Li H, et al. Aggravated restenosis and atherogenesis in ApoCIII transgenic mice but lack of protection in ApoCIII knockouts: the effect of authentic triglyceride-rich lipoproteins with and without ApoCIII. Cardiovasc Res. 2015;107(4):579-589.

49. Bomsztyk K, et al. PIXUL-ChIP: integrated high-throughput sample preparation and analytical platform for epigenetic studies. Nucleic Acids Res. 2019;47(12):e69.

50. Laatsch A, Merkel M, Talmud PJ, Grewal T, Beisiegel U, Heeren J. Insulin stimulates hepatic low density lipoprotein receptor-related protein 1 (LRP1) to increase postprandial lipoprotein clearance. Atherosclerosis. 2009;204(1):105-111.

51. Windler E, Havel RJ. Inhibitory effects of C apolipoproteins from rats and humans on the uptake of triglyceride-rich lipoproteins and their remnants by the perfused rat liver. J Lipid Res. 1985;26(5):556-565.

52. Aalto-Setälä K, et al. Mechanism of hypertriglyceridemia in human apolipoprotein (apo) CIII transgenic mice. Diminished very low density lipoprotein fractional catabolic rate associated with increased apo CIII and reduced apo $\mathrm{E}$ on the particles. JClin Invest. 1992;90(5):1889-1900.

53. Kowal RC, Herz J, Weisgraber KH, Mahley RW, Brown MS, Goldstein JL. Opposing effects of apolipoproteins $\mathrm{E}$ and $\mathrm{C}$ on lipoprotein binding to low density lipoprotein receptor-related protein. J Biol Chem. 1990;265(18):10771-10779.

54. Olin-Lewis K, et al. ApoC-III content of apoB-containing lipoproteins is associated with binding to the vascular proteoglycan biglycan. J Lipid Res. 2002;43(11):1969-1977.

55. Hiukka A, et al. ApoCIII-enriched LDL in type 2 diabetes displays altered lipid composition, increased susceptibility for sphingomyelinase, and increased binding to biglycan. Diabetes. 2009;58(9):2018-2026.

56. Dabelea D, et al. Effect of type 1 diabetes on the gender difference in coronary artery calcification: a role for insulin resistance? The Coronary Artery Calcification in Type 1 Diabetes (CACTI) Study. Diabetes. 2003;52(11):2833-2839.

57. Daugherty A, et al. Recommendation on design, execution, and reporting of animal atherosclerosis studies: a scientific statement from the American Heart Association. Arterioscler Thromb Vasc Biol. 2017;37(9):e131-e157.

58. Ronsein GE, et al. Parallel reaction monitoring (PRM) and selected reaction monitoring (SRM) exhibit comparable linearity, dynamic range and precision for targeted quantitative HDL proteomics. J Proteomics. 2015;113:388-399.

59. Shao B, et al. A cluster of proteins implicated in kidney disease is increased in high-density lipoprotein isolated from hemodialysis subjects. JProteome Res. 2015;14(7):2792-2806.

60. MacLean B, et al. Skyline: an open source document editor for creating and analyzing targeted proteomics experiments. Bioinformatics. 2010;26(7):966-968.

61. Basu A, et al. Serum apolipoproteins and apolipoprotein-defined lipoprotein subclasses: a hypothesis-generating prospective study of cardiovascular events in T1D. J Lipid Res. 2019;60(8):1432-1439. 